\title{
A SHARP SOBOLEV INEQUALITY ON RIEMANNIAN MANIFOLDS*
}

\author{
YANYAN LI \\ Department of Mathematics, Rutgers University \\ 110 Frelinghuysen Rd. Piscataway, NJ 08854-8019, U.S.A. \\ TONIA RICCIARDI \\ Departimento di Matematica e Applicazioni \\ Universitá di Napoli Federico II Via Cintia, 80126 Napoli, Italy
}

\begin{abstract}
Let $(M, g)$ be a smooth compact Riemannian manifold without boundary of dimension $n \geq 6$. We prove that

$$
\|u\|_{L^{2^{*}(M, g)}}^{2} \leq K^{2} \int_{M}\left\{\left|\nabla_{g} u\right|^{2}+c(n) R_{g} u^{2}\right\} d v_{g}+A\|u\|_{L^{2 n /(n+2)}(M, g)}^{2},
$$

for all $u \in H^{1}(M)$, where $2^{*}=2 n /(n-2), c(n)=(n-2) /[4(n-1)], R_{g}$ is the scalar curvature, $K^{-1}=\inf \|\nabla u\|_{L^{2}\left(\mathbb{R}^{n}\right)}\|u\|_{L^{2 n /(n-2)}\left(\mathbb{R}^{n}\right)}^{-1}$ and $A>0$ is a constant depending on $(M, g)$ only. The inequality is sharp in the sense that on any $(M, g), K$ can not be replaced by any smaller number and $R_{g}$ can not be replaced by any continuous function which is smaller than $R_{g}$ at some point. If $(M, g)$ is not locally conformally flat, the exponent $2 n /(n+2)$ can not be replaced by any smaller number. If $(M, g)$ is locally conformally flat, a stronger inequality, with $2 n /(n+2)$ replaced by 1 , holds in all dimensions $n \geq 3$.
\end{abstract}

1. Introduction. Considerable work has been devoted to the analysis of sharp Sobolev-type inequalities, very often in connection with concrete problems from geometry and physics. See, e.g., Trudinger [44], Moser [34], Aubin [3, 4], Talenti [43], Brezis and Nirenberg [10], Lieb [32], Lieb and Thirring [33], Carlen and Loss [14], Carleson and Chang [15], Struwe [42], Escobar [22], and Beckner [8].

In order to fix notation, we recall a classical result. For $n \geq 3$ and $2^{*}=2 n /(n-2)$, it was shown by Aubin [3] and Talenti [43] that

$$
K^{-1}=\inf \left\{\frac{\|\nabla u\|_{L^{2}\left(\mathbb{R}^{n}\right)}}{\|u\|_{L^{2^{*}}\left(\mathbb{R}^{n}\right)}}: u \in L^{2^{*}}\left(\mathbb{R}^{n}\right) \backslash\{0\},|\nabla u| \in L^{2}\left(\mathbb{R}^{n}\right)\right\},
$$

where $K^{2}=4 /\left[n(n-2) \sigma_{n}^{2 / n}\right]$ and where $\sigma_{n}$ is the volume of the standard $n$-sphere. They also showed that the infimum is attained and, modulo non-zero constant

1991 Mathematics Subject Classification. 35J60, 58E35.

Key words and phrases. sharp Sobolev inequality, critical exponent, Yamabe problem.

*Y.Y.L. was partially supported by National Science Foundation Grant DMS-0100819 and a Rutgers University Research Council Grant. T.R. was partially supported by CNR Fellowship 203.01.69 (19/01/98) and by PRIN 2000 "Variational Methods and Nonlinear Differential Equations". 
multiples, the set of minimizers is given by

$$
\left\{U_{y, \lambda} ; y \in \mathbb{R}^{n}, \lambda>0\right\},
$$

where

$$
\begin{aligned}
& U_{y, \lambda}(x)=\lambda^{(n-2) / 2} U(\lambda(x-y)), \\
& U(x)=U_{0,1}(x)=\left(\frac{1}{1+\bar{\lambda}^{2}|x|^{2}}\right)^{\frac{n-2}{2}}
\end{aligned}
$$

and $\bar{\lambda}^{2}=[n(n-2)]^{-1} K^{-2}$. The function $U$ is characterized as the unique solution of the equation

$$
-\Delta U=K^{-2} U^{2^{*}-1} \quad \text { in } \mathbb{R}^{n}
$$

satisfying

$$
\begin{aligned}
& U \in D^{1,2}\left(\mathbb{R}^{n}\right), \quad 0<U \leq 1, \\
& U(0)=1, \quad \int_{\mathbb{R}^{n}} U^{2^{*}} d x=1 .
\end{aligned}
$$

A conjecture was made by Aubin [3]: On any smooth compact Riemannian manifold $(M, g)$ of dimension $n \geq 3$, there exists a constant $A>0$ depending only on $(M, g)$, such that

$$
\|u\|_{L^{2^{*}}(M, g)}^{2} \leq K^{2}\left\|\nabla_{g} u\right\|_{L^{2}(M, g)}^{2}+A\|u\|_{L^{2}(M, g)}^{2}, \quad \forall u \in H^{1}(M) .
$$

The conjecture was proved in [3] for manifolds of constant sectional curvature. He also proved a weaker version of (1.3), where for any $\varepsilon>0, K$ is replaced by $K+\varepsilon$ and where $A$ is allowed to depend on $\varepsilon$.

Various related questions in bounded domains $\Omega$ of $\mathbb{R}^{n}$ have been extensively studied. In particular, the following result was proved by Brezis and Nirenberg [10]: For $n=3$, there exists a constant $\lambda^{*}>0$ such that

$$
\|\nabla u\|_{L^{2}(\Omega)}^{2} \geq K^{-2}\|u\|_{L^{6}(\Omega)}^{2}+\lambda^{*}\|u\|_{L^{2}(\Omega)}^{2}, \quad \forall u \in H_{0}^{1}(\Omega),
$$

where $\lambda^{*}$ depends on $\Omega$; when $\Omega$ is a ball, $\lambda^{*}$ can be taken as $\frac{1}{4} \pi^{2}(3|\Omega| /(4 \pi))^{-2 / 3}$ which is sharp. They also showed that for $n \geq 4$ and for all $q<n /(n-2)$,

$$
\|\nabla u\|_{L^{2}(\Omega)}^{2} \geq K^{-2}\|u\|_{L^{2^{*}}(\Omega)}^{2}+\lambda_{q}\|u\|_{L^{q}(\Omega)}^{2}, \quad \forall u \in H_{0}^{1}(\Omega),
$$

where $\lambda_{q}>0$ depends on $\Omega$ and $q$. On the other hand they pointed out that, on any $\Omega$, such an inequality can not hold with $q=n /(n-2)$. Further results were obtained by Brezis and Lieb [9], and closely related ones by Adimurthi and Yadava [1]. Results of similar nature, concerning the Hardy-Littlewood inequality for functions with support in a ball of $\mathbb{R}^{n}$, were obtained and used by Daubechies and Lieb [16]. We refer to Brezis and Marcus [11], Brezis, Marcus and Shafrir [12], and Shafrir [38] for more recent related works on the sharp Hardy-Littlewood inequality.

The conjecture (1.3) was proved by Hebey and Vaugon [27]. Results of similar nature for manifolds with boundary were established by $\mathrm{Li}$ and $\mathrm{Zhu}[30,31]$, with improvements given by Zhu $[45,46]$. A $W^{1, p}$ version of $(1.3)$ with $p \neq 2$, also conjectured by Aubin [3], was proved through the work of Aubin and Li [6], and Druet $[18,19]$. It should be mentioned that in two dimensions, the corresponding inequality discovered by Trudinger [44] has also been widely investigated and applied in its sharp form, due to Moser [34]. A sharp Moser-Trudinger inequality on Riemannian 2-manifolds was established by Fontana [23], and used by Gillet and Soule [25]. Alternative proofs of Fontana's result and connections to the analysis of 
vortices in the Chern-Simon-Higgs gauge theory were given by Ding, Jost, Li and Wang [17] and by Nolasco and Tarantello [35, 36].

Statement of the main results. Our main result in this paper is the following sharp Sobolev inequality on Riemannian manifolds of dimension $n \geq 6$ :

Theorem 1.1 (Main Result). Let $(M, g)$ be a smooth compact Riemannian manifold without boundary of dimension $n \geq 6$. There exists a constant $A>0$, depending on $(M, g)$ only, such that for all $u \in H^{1}(M)$ there holds:

$$
\|u\|_{L^{2^{*}}(M, g)}^{2} \leq K^{2} \int_{M}\left\{\left|\nabla_{g} u\right|^{2}+c(n) R_{g} u^{2}\right\} d v_{g}+A\|u\|_{L^{\bar{r}}(M, g)}^{2},
$$

where $2^{*}$ and $K$ are defined above, $c(n)=(n-2) /[4(n-1)], \bar{r}=2 n /(n+2)=2^{* \prime}$, $R_{g}$ is the scalar curvature of $g$.

We point out that our proof of Theorem 1.1 does not make any use of inequality (1.3), which on the other hand is an easy consequence.

REMARK 1.2 (Sharpness). Theorem 1.1 is sharp, in the sense that one can neither replace $K$ by any smaller number, nor replace $R_{g}$ by any $R_{g}+f$ with $f \in C^{0}$ negative somewhere. Moreover, if $(M, g)$ is not locally conformally flat, one cannot replace $\bar{r}$ by any smaller number.

The case of locally conformally flat manifolds is completely described by our next result:

THEOREM 1.3. Let $(M, g)$ be a smooth compact locally conformally flat Riemannian manifold without boundary of dimension $n \geq 3$. There exists a constant $A>0$, depending on $(M, g)$ only, such that for all $u \in H^{1}(M)$ there holds:

$$
\|u\|_{L^{2^{*}}(M, g)}^{2} \leq K^{2} \int_{M}\left\{\left|\nabla_{g} u\right|^{2}+c(n) R_{g} u^{2}\right\} d v_{g}+A\|u\|_{L^{1}(M, g)}^{2} .
$$

In view of the work of Schoen [39], we expect a positive answer to

QUESTION 1.4. For locally conformally flat manifolds and for manifolds of dimension $3 \leq n \leq 5$, are there some Sobolev type inequalities involving global geometric quantities?

For manifolds with positive total scalar curvature $\int_{M} R_{g} d v_{g}$, a natural global geometric quantity is the "mass", which corresponds to the leading term of the regular part of the Green's function for the conformal Laplacian, see [39, 28].

The sharpness of Theorem 1.1 as stated in Remark 1.2 can be deduced from the following expansions due to Aubin [4] (see also [5, 28]). Let $P \in M, \lambda>0$ and let $h$ be a Riemannian metric on $M$; denote by $\xi_{P, \lambda}^{h}$ the " $h$-bubble" defined for $x \in M$ by

$$
\xi_{P, \lambda}^{h}(x)=\left(\frac{\lambda}{1+(\lambda \bar{\lambda})^{2} \operatorname{dist}_{h}^{2}(x, P)}\right)^{\frac{n-2}{2}} .
$$

Let $\eta$ be a smooth cutoff function supported near $P$, and set $\widetilde{\xi}_{P, \lambda}^{h}=\eta \xi_{P, \lambda}^{h}$. Then, as $\lambda \rightarrow \infty$,

$$
Y_{h}\left(\widetilde{\xi}_{P, \lambda}^{h}\right)= \begin{cases}K^{-2}-\gamma_{n}\left|W_{h}(P)\right|^{2} \lambda^{-4}+\circ\left(\lambda^{-4}\right), & \text { if } n \geq 7, \\ K^{-2}-\gamma_{n}\left|W_{h}(P)\right|^{2} \lambda^{-4} \log \lambda+\circ\left(\lambda^{-4} \log \lambda\right), & \text { if } n=6,\end{cases}
$$


where $\gamma_{n}>0$ is a dimensional constant, $W_{h}(P)$ is the Weyl tensor of $h$ at $P$ and $Y_{h}$ denotes the Yamabe functional:

$$
Y_{h}(u)=\frac{\int_{M}\left\{\left|\nabla_{h} u\right|^{2}+c(n) R_{h} u^{2}\right\} d v_{h}}{\left(\int_{M}|u|^{2^{*}} d v_{h}\right)^{2 / 2^{*}}}, \quad u \in H^{1}(M) \backslash\{0\} .
$$

To see the sharpness of Theorem 1.1, we note that if $K$ is replaced by any smaller number, then (1.4) is violated by $u=\widetilde{\xi}_{P, \lambda}^{g}$ for large $\lambda$ (fixing any $P \in M$ ); if $R_{g}$ is replaced by $R_{g}+f$ with $f(\bar{P})<0$ for some $\bar{P} \in M$, then (1.4) is violated by $u=\widetilde{\xi}_{\bar{P}, \lambda}^{g}$ for large $\lambda$; if $\bar{r}$ is replaced by some $1 \leq s<\bar{r}$, then we have $\left\|\widetilde{\xi}_{P, \lambda}^{g}\right\|_{L^{s}(M)}=$ $\circ\left(\left\|\widetilde{\xi}_{P, \lambda}\right\|_{L_{\bar{r}}(M)}\right)$, and thus inequality (1.4) and (1.6)-(1.7) imply that $\left|W_{g}(P)\right|=0$ for all $P \in M$, i.e., $(M, g)$ is locally conformally flat.

In view of (1.6) and our results we expect a positive answer to

QUESTION 1.5. Are there some refined versions of (1.4) involving the Weyl tensor?

Outline of the proofs. We first sketch the proof of Theorem 1.3, which is simple, and relies on a "local to global" argument, given in the Appendix. By a local to global argument, we mean that we first establish the inequality for all functions $u \in H^{1}(M)$ supported in a ball of fixed diameter $\varepsilon>0$, and then we extend the inequality to arbitrary $u \in H^{1}(M)$. Another ingredient is the following well-known transformation property of the conformal Laplacian, see, e.g., [40]:

$$
-\Delta_{\widehat{h}} u+c(n) R_{\widehat{h}} u=\varphi^{1-2^{*}}\left\{-\Delta_{h}(u \varphi)+c(n) R_{h}(u \varphi)\right\},
$$

for all $u \in H^{1}(M)$, where $\widehat{h}=\varphi^{4 /(n-2)} h, \varphi \in C^{\infty}(M), \varphi>0$.

Proof of Theorem 1.3. Since $(M, g)$ is locally conformally flat, for some $\varepsilon>0$ independent of $P \in M$, we have $\left(B_{\varepsilon}(P), g\right) \cong\left(B, \varphi^{4 /(n-2)} \mathcal{E}\right)$, for some $\varphi>0$ (under control), with $\mathcal{E}$ the Euclidean metric. Since

$$
\left(\int_{B}|u|^{2^{*}} d x\right)^{2 / 2^{*}} \leq K^{2} \int_{B}|\nabla u|^{2} d x, \quad \forall u \in H_{0}^{1}(B),
$$

we have by (1.8),

$$
\left(\int_{B_{\varepsilon}(P)}|u|^{2^{*}} d v_{g}\right)^{2 / 2^{*}} \leq K^{2} \int_{B_{\varepsilon}(P)}\left\{\left|\nabla_{g} u\right|^{2}+c(n) R_{g} u^{2}\right\} d v_{g}, \quad \forall u \in H_{0}^{1}\left(B_{\varepsilon}(P)\right) .
$$

Now Theorem 1.3 follows from the above and from Lemma 7.1 in the Appendix.

The "local to global" approach has been systematically used by Aubin [3], Hebey and Vaugon [27], Aubin and Li [6], Druet, Hebey and Vaugon [20], and others. In $[30,31], \mathrm{Li}$ and Zhu introduced a global approach by attacking the problem directly on the whole manifold. Such an approach should be useful in obtaining a positive answer to Question 1.4, since the inequality would involve global quantities and therefore could not be obtained by a local to global approach.

We shall now provide a brief sketch of the proof of Theorem 1.1, which will occupy the main part of this paper. For simplicity of exposition, we shall restrict ourselves in the present sketch to the case $n \geq 7$. We argue by contradiction, and we take a global approach. Namely, for all $\alpha>0$ we define:

$$
I_{\alpha}(u)=\frac{\int_{M}\left\{\left|\nabla_{g} u\right|^{2}+c(n) R_{g} u^{2}\right\} d v_{g}+\alpha\|u\|_{L^{\bar{r}}(M, g)}^{2}}{\|u\|_{L^{2^{*}}(M, g)}^{2}}, \quad u \in H^{1}(M) \backslash\{0\} .
$$


Negating (1.4), we assume that

$$
\inf _{H^{1}(M) \backslash\{0\}} I_{\alpha}<K^{-2}, \quad \forall \alpha>0 .
$$

It is straightforward to check that inequality (1.4) holds for the family $\left\{t \widetilde{\xi}_{P, \lambda}^{g}\right\}$ defined above, uniformly in $t>0, P \in M, \lambda>0$. The underlying idea of the proof is that if (1.9) holds for all $\alpha>0$, then for all $\alpha>0$ there exist minimizers $u_{\alpha}$ of $I_{\alpha}$, which approach $\left\{t \widetilde{\xi}_{P, \lambda}^{g}\right\}$ as $\alpha \rightarrow+\infty$, and the convergence rate is sufficiently rapid to ensure that for some suitable $A>0, u_{\alpha}$ also satisfies (1.4), uniformly in $\alpha$. But then $\alpha \leq C$, a contradiction.

In Section 2, for the reader's convenience, we establish some preliminary results by suitably adapting to our needs some well-known techniques from $[44,4,10,27$, 6]. We show that (1.9) implies the existence of a minimizer $u_{\alpha} \in H^{1}(M)$ for $I_{\alpha}$ satisfying $u_{\alpha} \in H^{1}(M), u_{\alpha} \geq 0, \int_{M} u_{\alpha}^{2^{*}} d v_{g}=1$ and such that

$$
\mu_{\alpha}^{(n-2) / 2}:=\max _{M} u_{\alpha}^{-1}=: u_{\alpha}\left(x_{\alpha}\right)^{-1} \rightarrow 0 .
$$

We fix some small $\delta_{0}>0$ which depends only on $(M, g)$. We show:

$$
\begin{aligned}
& \left\|\nabla_{g}\left(u_{\alpha}-\xi_{x_{\alpha}, \mu_{\alpha}^{-1}}^{g}\right)\right\|_{L^{2}\left(B_{\delta_{0}}\right)}+\left\|u_{\alpha}-\xi_{x_{\alpha}, \mu_{\alpha}^{-1}}^{g}\right\|_{\left.L^{2^{*}\left(B_{\delta_{0}}\right.}\right)} \rightarrow 0 \\
& \mu_{\alpha}^{(n-2) / 2} u_{\alpha}\left(\exp _{x_{\alpha}}^{g}\left(\mu_{\alpha} \cdot\right)\right) \rightarrow U \quad \text { in } C_{\mathrm{loc}}^{2}\left(\mathbb{R}^{n}\right) .
\end{aligned}
$$

The $C_{\mathrm{loc}}^{2}\left(\mathbb{R}^{n}\right)$-convergence and a change of variables imply the lower bound:

$$
\left\|u_{\alpha}\right\|_{L^{\bar{r}}(M, g)} \geq C^{-1} \mu_{\alpha}^{2} .
$$

In Section 3 we prove the uniform estimate:

$$
u_{\alpha}(x) \leq C \mu_{\alpha}^{(n-2) / 2} \operatorname{dist}_{g}\left(x, x_{\alpha}\right)^{2-n}, \quad \forall x \in M .
$$

This estimate ensures a suitable decay of $u_{\alpha}$ away from $x_{\alpha}$; it is a key step. We note that pointwise estimates for minimizers to critical exponent equations have been established and used by Brezis and Peletier [13], Atkinson and Peletier [2], Rey [37], Han [26], Hebey and Vaugon [27], Li and Zhu [30, 31], Aubin and Li [6], and others. We derive our pointwise estimate along the line of [30, 31], by working directly on $u_{\alpha}$; new ingredients are needed in deriving our estimate.

In Section 4, in order to simplify calculations, we introduce a conformal metric $\widehat{g}=\psi^{4 /(n-2)} g$, with $\psi \in C^{\infty}(M), \psi\left(x_{\alpha}\right)=1, \frac{1}{2} \leq \psi \leq 2,\|\psi\|_{C^{2}} \leq C$, such that $R_{\widehat{g}} \equiv 0$ in $B_{\delta_{0}}\left(x_{\alpha}\right)$. Our pointwise estimates in Section 2 allow us to adapt ideas of Bahri and Coron [7] to make an energy estimate of the difference: $u_{\alpha} / \psi-t_{\alpha} \xi_{\tilde{x}_{\alpha}, \lambda_{\alpha}}^{\widehat{g}}$ in a small ball $B_{\delta_{\alpha}}\left(x_{\alpha}\right)$, where $\delta_{\alpha} \in\left[\delta_{0} / 2, \delta_{0}\right], t_{\alpha}>0, \mu_{\alpha}^{-1}\left|\tilde{x}_{\alpha}-x_{\alpha}\right| \rightarrow 0, \lambda_{\alpha}>0$ are "optimal" in a suitable sense. The main result of Section 4 is the estimate for the projection $u_{\alpha} / \psi-t_{\alpha} \xi_{\tilde{x}_{\alpha}, \lambda_{\alpha}}^{\widehat{g}}$ on $H_{0}^{1}\left(B_{\delta_{\alpha}}\left(x_{\alpha}\right)\right)$, denoted $w_{\alpha}$, as in Proposition 4.1.

In Section 5 we show that by choosing a "good radius" $\delta_{\alpha} \in\left[\delta_{0} / 2, \delta_{0}\right]$, the "boundary part" of $u_{\alpha} / \psi-t_{\alpha} \xi_{\tilde{x}_{\alpha}, \lambda_{\alpha}}^{\widehat{g}}$ may be controlled in $H^{1}\left(\partial B_{\alpha}\right)$, see Lemma 5.3. For $n \geq 7$, the estimate resulting from our pointwise estimates, Proposition 4.1 , Lemma 5.3 and taking into account (2.10) is given by:

$$
\left\|\nabla_{\widehat{g}}\left(\frac{u_{\alpha}}{\psi}-t_{\alpha} \xi_{\tilde{x}_{\alpha}, \lambda_{\alpha}}^{\widehat{g}}\right)\right\|_{L^{2}\left(B_{\delta_{\alpha}}\left(x_{\alpha}\right)\right)} \leq C\left(\mu_{\alpha}^{2}+\left(1+\mu_{\alpha}^{-2+\beta}\right) \alpha\left\|u_{\alpha}\right\|_{L^{\bar{r}}(M, g)}^{2}\right),
$$


where $\beta=(n-6)(n-2) /[2(n+2)]>0$ is strictly positive, since $n \geq 7$. By carefully exploiting orthogonality, we prove the following lower bound:

$$
Y_{g}\left(u_{\alpha}\right) \geq Y_{\widehat{g}}\left(\widetilde{\xi}_{\tilde{x}_{\alpha}, \lambda_{\alpha}}^{\widehat{g}}\right)+O\left(\mu_{\alpha}^{2}\left\|\nabla_{\widehat{g}}\left(\frac{u_{\alpha}}{\psi}-t_{\alpha} \xi_{\tilde{x}_{\alpha} \lambda_{\alpha}}^{\widehat{\widehat{a}}}\right)\right\|_{L^{2}\left(B_{\delta_{\alpha}}\left(x_{\alpha}\right)\right)}+\mu_{\alpha}^{n-2}\right),
$$

see Proposition 5.1.

At this point we have all the necessary ingredients to conclude the proof in the case $n \geq 7$. We note that the contradiction assumption (1.9) implies:

$$
K^{-2}>I_{\alpha}\left(u_{\alpha}\right)=Y_{g}\left(u_{\alpha}\right)+\alpha\left\|u_{\alpha}\right\|_{L^{\bar{r}}(M, g)}^{2} .
$$

By the above inequality and (1.12), we obtain

$$
\begin{aligned}
\alpha\left\|u_{\alpha}\right\|_{L^{\bar{r}}(M, g)}^{2} \leq & K^{-2}-Y_{\widehat{g}}\left(\widetilde{\xi}_{\tilde{x}_{\alpha}, \lambda_{\alpha}}^{\widehat{g}}\right) \\
& +C\left(\mu_{\alpha}^{2}\left\|\nabla_{\widehat{g}}\left(\frac{u_{\alpha}}{\psi}-t_{\alpha} \xi_{\tilde{x}_{\alpha}, \lambda_{\alpha}}^{\widehat{g}}\right)\right\|_{L^{2}\left(B_{\delta_{\alpha}}\left(x_{\alpha}\right)\right)}+\mu_{\alpha}^{n-2}\right) .
\end{aligned}
$$

By (1.6) (or an easy calculation since we do not need the explicit coefficient of $\lambda^{-4}$ ),

$$
\left|K^{-2}-Y_{\widehat{g}}\left(\widetilde{\xi}_{\tilde{x}_{\alpha}, \lambda_{\alpha}}^{\underline{g}}\right)\right| \leq C \mu_{\alpha}^{4} .
$$

Inserting (1.11) and (1.14) into (1.13), and recalling that $\beta>0$, we derive

$$
(1+\circ(1)) \alpha\left\|u_{\alpha}\right\|_{L^{\bar{r}}(M, g)}^{2} \leq C \mu_{\alpha}^{4} .
$$

In view of (1.10), the desired contradiction $\alpha \leq C$ follows, and Theorem 1.1 is established.

Finally, Section 6 is devoted to the proof of Theorem 1.1 in the remaining case $n=6$. This is more delicate than the case $n \geq 7$. Nevertheless, we can still obtain the inequality (1.4) with the aid of a uniform lower bound, reminiscent of an argument in [30].

Notation. Henceforth, $C>0$ always denotes a general constant independent of $\alpha$, and subsequences of $\alpha \rightarrow+\infty$ are taken without further notice. Denoting by $(\Omega, h)$ a Riemannian manifold (possibly with boundary), we set

$$
\begin{aligned}
& \langle\varphi, \psi\rangle_{h}=\int_{\Omega} \nabla_{h} \varphi \cdot \nabla_{h} \psi d v_{h}=\int_{\Omega} h^{i j} \frac{\partial \varphi}{\partial x^{i}} \frac{\partial \varphi}{\partial x^{j}} d v_{h}, \quad \forall \varphi, \psi \in H_{0}^{1}(\Omega), \\
& \|\varphi\|_{h}=\sqrt{\langle\varphi, \varphi\rangle_{h}}, \quad \forall \varphi \in H_{0}^{1}(\Omega) .
\end{aligned}
$$

We note that the metrics $g$ and $\widehat{g}$ defined above are both equivalent to the Euclidean metric $\mathcal{E}$. When the specific metric is clear from the context, or irrelevant up to equivalence to $g$, we do not indicate it explicitly. Furthermore, for $q \geq 1$ we denote:

$$
\begin{aligned}
& \|\varphi\|_{q}=\|\varphi\|_{L^{q}\left(B_{\alpha}\right)} \\
& \left\|U^{q}\right\|_{2^{* \prime}, \mu_{\alpha}^{-1}}=\left(\int_{B_{\mu_{\alpha}^{-1}}(0)} U^{2^{*^{\prime} q}} d y\right)^{1 / 2^{* \prime}}, \quad 2^{*^{\prime}}=\frac{2 n}{n+2},
\end{aligned}
$$

where $U$ is the standard minimizer on $\mathbb{R}^{n}$ defined above.

For ease of future reference, we prove our estimates for $n \geq 3$. Moreover, we obtain our estimates for a general exponent $r \in(1,2)$, which could even depend on $\alpha$ (this will also be convenient for the local to global argument sketched in the Appendix). The actual value $r=\bar{r}=2 n /(n+2)$ and the condition $n \geq 6$ are used only in the final part of the proof of Theorem 1.1, in Section 5 and in Section 6. 
Theorem 1.1 in the case $n \geq 7$ has been presented at the 966th AMS Meeting at Hoboken, NJ, April 28-29, 2001. The results in the present paper were announced in $[29]$.

2. Preliminaries. The preliminary results in this section are obtained by adapting standard methods to our situation, see, e.g., [44, 4, 10, 27, 6]. For the reader's convenience, we sketch their proofs. Throughout this section, we assume $n \geq 3$.

For every $\alpha>0$ and for $r \in(1,2)$ (possibly depending on $\alpha$ ) we consider the functional:

$$
I_{\alpha}(u)=\frac{\int_{M}\left\{\left|\nabla_{g} u\right|^{2}+c(n) R_{g} u^{2}\right\} d v_{g}+\alpha\|u\|_{L^{r}(M)}^{2}}{\|u\|_{L^{2^{*}}(M)}^{2}},
$$

defined for all $u \in H^{1}(M) \backslash\{0\}$. If (1.4) is false, then for all $\alpha>0$ we have

$$
\inf _{H^{1}(M) \backslash\{0\}} I_{\alpha}<K^{-2} .
$$

Proposition 2.1 (Existence of a minimizer). For all $\alpha>0$ there exists a nonnegative minimizer $u_{\alpha} \in H^{1}(M)$ such that

$$
\begin{aligned}
& I_{\alpha}\left(u_{\alpha}\right)=\ell_{\alpha}=\inf _{H^{1}(M) \backslash\{0\}} I_{\alpha}<K^{-2}, \\
& \int_{M} u_{\alpha}^{2^{*}} d v_{g}=1 .
\end{aligned}
$$

Moreover, $u_{\alpha} \in C^{2, r-1}(M)$ is a classical solution of the Euler-Lagrange equation:

$$
-\Delta_{g} u_{\alpha}+c(n) R_{g} u_{\alpha}+\alpha\left\|u_{\alpha}\right\|_{L^{r}(M)}^{2-r} u_{\alpha}^{r-1}=\ell_{\alpha} u_{\alpha}^{2^{*}-1} \quad \text { on } M .
$$

Proof. By homogeneity, it is equivalent to minimize $I_{\alpha}$ on the set

$$
\mathcal{A}=\left\{u \in H^{1}(M): \int_{M}|u|^{2^{*}} d v_{g}=1\right\} .
$$

However, $\mathcal{A}$ is not sequentially weakly closed in $H^{1}(M)$. Therefore, as usual, for fixed $\alpha$ and for all $1 \leq q<2^{*}$ we define:

$$
\mathcal{A}_{q}=\left\{u \in H^{1}(M): \int_{M}|u|^{q} d v_{g}=1\right\}
$$

and we consider the functional

$$
I_{q}(u)=\frac{\int_{M}\left\{\left|\nabla_{g} u\right|^{2}+c(n) R_{g} u^{2}\right\} d v_{g}+\alpha\left(\int_{M}|u|^{r} d v_{g}\right)^{2 / r}}{\left(\int_{M}|u|^{q} d v_{g}\right)^{2 / q}}
$$

on $\mathcal{A}_{q}$. By standard arguments $\inf _{\mathcal{A}_{q}} I_{q}$ is attained, i.e., for every $1 \leq q<2^{*}$ there exists $u_{q} \in \mathcal{A}_{q}$ such that

$$
I_{q}\left(u_{q}\right)=\inf _{\mathcal{A}_{q}} I_{q}=: \ell_{q}
$$

The minimizer $u_{q}$ satisfies the Euler-Lagrange equation:

$$
-\Delta_{g} u_{q}+c(n) R_{g} u_{q}+\alpha\left\|u_{q}\right\|_{L^{r}(M)}^{2-r} u_{q}^{r-1}=\ell_{q} u_{q}^{q-1} \quad \text { on } M .
$$

The sequence $u_{q}$ is bounded in $H^{1}(M)$; therefore passing to a subsequence we can assume that there exists $u_{\alpha} \in H^{1}(M)$ such that $u_{q} \rightarrow u_{\alpha}$ weakly in $H^{1}(M)$, strongly in $L^{2}(M)$ and a.e. Since for every fixed $u$ we have $I_{q}(u) \rightarrow I_{\alpha}(u)$ as $q \rightarrow 2^{*}$, it is clear that

$$
\limsup _{q \rightarrow \infty} \ell_{q} \leq \ell_{\alpha}<K^{-2}
$$


Consequently, for every $0<2^{*}-q \ll 1$, we can apply the Moser iteration technique to (2.3) to derive a uniform bound $\sup _{M} u_{q} \leq C(\alpha)$, where $C(\alpha)>0$ is a constant independent of $q$ (see, e.g., [6]). Then by dominated convergence, $u_{\alpha} \in \mathcal{A}$ and by weak semicontinuity $I_{\alpha}\left(u_{\alpha}\right) \leq \liminf \ell_{q} \leq \ell_{\alpha}$. The $u_{\alpha}$ is a desired minimizer.

The proof of the existence of the minimizer shows that $u_{\alpha}$ is in $L^{\infty}(M)$ for every fixed $\alpha$. Then standard elliptic theory implies that $u_{\alpha} \in C^{1, \beta}(M)$ for some $0<\beta<$ 1. Therefore $u_{\alpha}^{r-1} \in C^{0, r-1}(M)$, and by Schauder estimates $u_{\alpha} \in C^{2, r-1}(M)$.

REMARK 2.2. Since $0<r-1<1$, the nonlinearity $u^{r-1}$ is sublinear and therefore we can not use the maximum principle to conclude $u_{\alpha}>0$ on $M$.

Proposition 2.3 (Standard blowup). As $\alpha \rightarrow+\infty$, we have:

$$
\begin{aligned}
& u_{\alpha} \rightarrow 0, \text { weakly in } H^{1}(M), \text { strongly in } L^{p}(M) \forall 1 \leq p<2^{*} \text { and a.e., } \\
& \int_{M}\left|\nabla_{g} u_{\alpha}\right|^{2} d v_{g} \rightarrow K^{-2}, \\
& \alpha\left\|u_{\alpha}\right\|_{L^{r}(M)}^{2} \rightarrow 0, \\
& \ell_{\alpha} \rightarrow K^{-2} \\
& \max _{M} u_{\alpha} \rightarrow+\infty .
\end{aligned}
$$

Proof. By compactness, for any $\varepsilon>0$ there exists $C_{\varepsilon}>0$ such that:

$$
\|u\|_{L^{2}(M)}^{2} \leq \varepsilon \int_{M}\left|\nabla_{g} u\right|^{2} d v_{g}+C_{\varepsilon}\|u\|_{L^{r}(M)}^{2} .
$$

So,

$$
\begin{aligned}
I_{\alpha}\left(u_{\alpha}\right) & =\ell_{\alpha}=\int_{M}\left\{\left|\nabla_{g} u_{\alpha}\right|^{2}+c(n) R_{g} u_{\alpha}^{2}\right\} d v_{g}+\alpha\left\|u_{\alpha}\right\|_{L^{r}(M)}^{2} \\
& \geq\left(1-\varepsilon c(n) \max _{M}\left|R_{g}\right|\right) \int_{M}\left|\nabla_{g} u_{\alpha}\right|^{2} d v_{g}+\left(\alpha-C_{\varepsilon}\right)\left\|u_{\alpha}\right\|_{L^{r}(M)}^{2} .
\end{aligned}
$$

Fixing a small $\varepsilon$ we obtain:

$$
\frac{1}{2} \int_{M}\left|\nabla_{g} u_{\alpha}\right|^{2} d v_{g}+\left(\alpha-C_{\varepsilon}\right)\left\|u_{\alpha}\right\|_{L^{r}(M)}^{2} \leq I_{\alpha}\left(u_{\alpha}\right)<K^{-2} .
$$

Consequently,

and therefore,

$$
\int_{M}\left|\nabla_{g} u_{\alpha}\right|^{2} d v_{g} \leq C, \quad \alpha\left\|u_{\alpha}\right\|_{L^{r}(M)}^{2} \leq C
$$

$$
\int_{M} u_{\alpha}^{r} d v_{g} \rightarrow 0 \quad \text { as } \alpha \rightarrow+\infty
$$

Passing to a subsequence, we have (i). Furthermore, we can assume that for some $\theta, \eta \in[0,+\infty)$ there holds (along a subsequence):

$$
\int_{M}\left|\nabla_{g} u_{\alpha}\right|^{2} d v_{g} \rightarrow \theta \quad \text { and } \quad \alpha\left\|u_{\alpha}\right\|_{L^{r}(M)}^{2} \rightarrow \eta, \quad \text { as } \alpha \rightarrow+\infty .
$$

Proof of (ii)-(iii). We have to show that $\theta=K^{-2}$ and $\eta=0$. By the Sobolev inequality as in [5], for every $\varepsilon>0$ there exists $A_{\varepsilon}>0$ such that:

$$
\left\|u_{\alpha}\right\|_{L^{2^{*}(M)}}^{2} \leq K^{2}(1+\varepsilon) \int_{M}\left|\nabla_{g} u_{\alpha}\right|^{2} d v_{g}+A_{\varepsilon}\left\|u_{\alpha}\right\|_{L^{r}(M)}^{2} .
$$


Letting $\alpha \rightarrow+\infty$ in (2.4) we obtain:

$$
1=\left(\int_{M} u_{\alpha}^{2^{*}} d v_{g}\right)^{2 / 2^{*}} \leq K^{2}(1+\varepsilon) \theta
$$

Sending $\varepsilon \rightarrow 0$, we conclude $1 \leq K^{2} \theta$. On the other hand, we have by definition of $u_{\alpha}$ :

$$
\int_{M}\left\{\left|\nabla_{g} u_{\alpha}\right|^{2}+c(n) R_{g} u_{\alpha}^{2}\right\} d v_{g}+\alpha\left\|u_{\alpha}\right\|_{L^{r}(M)}^{2}=\ell_{\alpha}<K^{-2}
$$

Sending $\alpha \rightarrow+\infty$ we find $\theta+\eta \leq K^{-2}$. It follows that $\theta=K^{-2}$ and $\eta=0$, as asserted.

Proof of (iv). This is an immediate consequence of (i)-(ii)-(iii) and the definition of $\ell_{\alpha}$.

Proof of (v). We have:

$$
1=\int_{M} u_{\alpha}^{2^{*}} d v_{g} \leq\left(\max _{M} u_{\alpha}\right)^{2^{*}-r} \int_{M} u_{\alpha}^{r} d v_{g}=\circ(1)\left(\max _{M} u_{\alpha}\right)^{2^{*}-r} .
$$

Our next aim is to show that, after rescaling, the limit profile of $u_{\alpha}$ is the standard minimizer $U$, and that $u_{\alpha}$ approaches this limit "in energy", as in Proposition 2.4. Let $x_{\alpha} \in M$ be a maximum point of $u_{\alpha}$, namely $u_{\alpha}\left(x_{\alpha}\right)=\max _{M} u_{\alpha}$, then by Proposition 2.3-(v) we have

$$
\mu_{\alpha}:=u_{\alpha}\left(x_{\alpha}\right)^{-2 /(n-2)} \rightarrow 0 \quad \text { as } \alpha \rightarrow+\infty .
$$

Let $\delta_{0}>0$ be a small constant to be fixed below (e.g., less than injectivity radius). Let $\delta_{0} / 2 \leq \delta_{\alpha} \leq \delta_{0}$.

Proposition 2.4 (Convergence in energy).

$$
\lim _{\alpha \rightarrow+\infty} \int_{B_{\delta_{\alpha}}\left(x_{\alpha}\right)}\left\{\left|\nabla_{g}\left(u_{\alpha}-\xi_{x_{\alpha}, \mu_{\alpha}^{-1}}^{g}\right)\right|^{2}+\left|u_{\alpha}-\xi_{x_{\alpha}, \mu_{\alpha}^{-1}}^{g}\right|^{2^{*}}\right\} d v_{g}=0 .
$$

Proof. We consider the following rescaling of $u_{\alpha}$ on the geodesic ball $B_{\delta_{\alpha}}\left(x_{\alpha}\right)$ :

$$
v_{\alpha}(y)=\mu_{\alpha}^{(n-2) / 2} u_{\alpha}\left(\exp _{x_{\alpha}}\left(\mu_{\alpha} y\right)\right), \quad y \in \Omega_{\alpha},
$$

where

$$
\Omega_{\alpha}=\mu_{\alpha}^{-1} \exp _{x_{\alpha}}^{-1}\left(B_{\delta_{\alpha}}\left(x_{\alpha}\right)\right)=\mu_{\alpha}^{-1} B_{\delta_{\alpha}}(0)
$$

$v_{\alpha}$ satisfies

$$
-\Delta_{g_{\alpha}} v_{\alpha}+c(n) R_{g_{\alpha}} v_{\alpha}+\varepsilon_{\alpha} v_{\alpha}^{r-1}=\ell_{\alpha} v_{\alpha}^{2^{*}-1} \quad \text { in } \Omega_{\alpha}
$$

where

and

$$
g_{\alpha}(y)=g\left(\exp _{x_{\alpha}}\left(\mu_{\alpha} y\right)\right), \quad\left|R_{g_{\alpha}}\right| \leq C \mu_{\alpha}^{2},
$$

$$
\varepsilon_{\alpha}:=\alpha \mu_{\alpha}^{n-\frac{n-2}{2} r}\left\|u_{\alpha}\right\|_{L^{r}(M)}^{2-r} .
$$

We observe that the rescaled metric $g_{\alpha}$ converges to the Euclidean metric $\left(\delta_{i j}\right)$ on $\mathbb{R}^{n}$ uniformly on compact subsets, and it is equivalent to $\left(\delta_{i j}\right)$, uniformly in $\alpha$, i.e., there exists $C>0$ independent of $\alpha$ such that $C^{-1} \delta_{i j} \leq g_{\alpha, i j}(y) \leq C \delta_{i j}$. We claim that

$$
\varepsilon_{\alpha} \leq \alpha\left\|u_{\alpha}\right\|_{L^{r}(M)}^{2} \rightarrow 0 \quad \text { as } \alpha \rightarrow+\infty
$$


Indeed, by the definition of $\mu_{\alpha}$ and $\varepsilon_{\alpha}$,

$$
\varepsilon_{\alpha}=\frac{\alpha\left\|u_{\alpha}\right\|_{L^{r}(M)}^{2}}{\left(\max _{M} u_{\alpha}\right)^{2^{*}-r} \int_{M} u_{\alpha}^{r} d v_{g}}
$$

and

$$
1=\int_{M} u_{\alpha}^{2^{*}} d v_{g} \leq\left(\max _{M} u_{\alpha}\right)^{2^{*}-r} \int_{M} u_{\alpha}^{r} d v_{g} .
$$

Property (2.10) now follows by Proposition 2.3-(iii). By a change of variables,

$$
\int_{\Omega_{\alpha}} v_{\alpha}^{2^{*}} d v_{g_{\alpha}}=\int_{B_{\alpha}} u_{\alpha}^{2^{*}} d v_{g}
$$

and

$$
\int_{\Omega_{\alpha}}\left|\nabla_{g_{\alpha}} v_{\alpha}\right|^{2} d v_{g_{\alpha}}=\int_{B_{\alpha}}\left\{\left|\nabla_{g} u_{\alpha}\right|^{2}+c(n) R_{g} u_{\alpha}^{2}\right\} d v_{g} .
$$

Consequently, by the definition of $u_{\alpha}$

$$
\limsup _{\alpha \rightarrow+\infty} \int_{\Omega_{\alpha}} v_{\alpha}^{2^{*}} d v_{g_{\alpha}} \leq 1
$$

and by Proposition 2.3-(i)-(ii),

$$
\limsup _{\alpha \rightarrow+\infty} \int_{\Omega_{\alpha}}\left|\nabla_{g_{\alpha}} v_{\alpha}\right|^{2} d v_{g_{\alpha}} \leq K^{-2}
$$

By the definition of $\mu_{\alpha}, v_{\alpha}(y) \leq v_{\alpha}(0)=1$, thus, by standard elliptic estimates, there exists $v \in C_{\text {loc }}^{1}\left(\mathbb{R}^{n}\right)$ such that, along a subsequence, $v_{\alpha} \rightarrow v$ in $C_{\text {loc }}^{1}\left(\mathbb{R}^{n}\right)$, and $v(0)=1$. Furthermore, $v$ satisfies:

$$
\int_{\mathbb{R}^{n}}|\nabla v|^{2} d y=\lim _{R \rightarrow+\infty} \int_{B_{R}}|\nabla v|^{2} d y=\lim _{R \rightarrow+\infty} \lim _{\alpha \rightarrow+\infty} \int_{B_{R}}\left|\nabla_{g_{\alpha}} v_{\alpha}\right|^{2} d v_{g_{\alpha}} \leq K^{-2},
$$

and

$$
\int_{\mathbb{R}^{n}} v^{2^{*}} d y=\lim _{R \rightarrow+\infty} \int_{B_{R}} v^{2^{*}} d y=\lim _{R \rightarrow+\infty} \lim _{\alpha \rightarrow+\infty} \int_{B_{R}} v_{\alpha}^{2^{*}} d v_{g_{\alpha}} \leq 1
$$

In particular, $v \in D^{1,2}\left(\mathbb{R}^{n}\right)$, and taking pointwise limits in (2.9) we find that $v$ satisfies:

$$
\begin{aligned}
& -\Delta v=K^{-2} v^{2^{*}-1}, \quad \text { in } \mathbb{R}^{n} \\
& 0 \leq v \leq 1, v(0)=1 .
\end{aligned}
$$

Multiplying the above equation by $v$ and integrating by parts, and recalling the definition of $K$ we have:

$$
K^{-2} \int_{\mathbb{R}^{n}} v^{2^{*}} d y=\int_{\mathbb{R}^{n}}|\nabla v|^{2} d y \geq K^{-2}\left(\int_{\mathbb{R}^{n}} v^{2^{*}} d y\right)^{2 / 2^{*}} .
$$

Therefore, $\left(\int_{\mathbb{R}^{n}} v^{2^{*}} d y\right)^{1-2 / 2^{*}} \geq 1$, which together with (2.13) and (2.14) implies $\int_{\mathbb{R}^{n}} v^{2^{*}} d y=1$ and $\int_{\mathbb{R}^{n}}|\nabla v|^{2} d y=K^{-2}$ and thus necessarily $v=U$. Since the limit $v$ is independent of subsequences, the convergence is for all $\alpha \rightarrow+\infty$ with $x_{\alpha} \rightarrow P$. At this point, it is intuitively clear that Proposition 2.3-(ii) should imply the "strong convergence" (2.6); however we face some minor technicality due to the 
fact that $v_{\alpha}$ does not necessarily vanish on $\partial \Omega_{\alpha}$. Using the elementary calculus inequality:

$$
|| a+\left.b\right|^{p}-|a|^{p}-|b|^{p} \mid \leq C(p)\left(|a|^{p-1}|b|+|a||b|^{p-1}\right), \quad \forall a, b \in \mathbb{R}^{n}, p \geq 1
$$

with $p=2^{*}, a=U$, and $b=v_{\alpha}-U$, we have:

$$
\begin{array}{rl}
\int_{\Omega_{\alpha}}\left|v_{\alpha}-U\right|^{2^{*}} & d v_{g_{\alpha}} \leq \int_{\Omega_{\alpha}} v_{\alpha}^{2^{*}} d v_{g_{\alpha}}-\int_{\Omega_{\alpha}} U^{2^{*}} d v_{g_{\alpha}} \\
& +C\left(\int_{\Omega_{\alpha}} U^{2^{*}-1}\left|v_{\alpha}-U\right| d v_{g_{\alpha}}+\int_{\Omega_{\alpha}} U\left|v_{\alpha}-U\right|^{2^{*}-1} d v_{g_{\alpha}}\right) \\
\leq & \circ(1)+C\left(\int_{\Omega_{\alpha}} U^{2^{*}-1}\left|v_{\alpha}-U\right| d v_{g_{\alpha}}+\int_{\Omega_{\alpha}} U\left|v_{\alpha}-U\right|^{2^{*}-1} d v_{g_{\alpha}}\right) .
\end{array}
$$

The right hand side is easily seen to vanish as $\alpha \rightarrow+\infty$ :

$$
\begin{aligned}
& \int_{\Omega_{\alpha}} U\left|v_{\alpha}-U\right|^{2^{*}-1} d v_{g_{\alpha}} \\
= & \int_{B_{R}} U\left|v_{\alpha}-U\right|^{2^{*}-1} d v_{g_{\alpha}}+\int_{\Omega_{\alpha} \backslash B_{R}} U\left|v_{\alpha}-U\right|^{2^{*}-1} d v_{g_{\alpha}} \\
\leq & \int_{B_{R}} U\left|v_{\alpha}-U\right|^{2^{*}-1} d v_{g_{\alpha}} \\
& \quad+\left(\int_{\Omega_{\alpha} \backslash B_{R}} U^{2^{*}} d v_{g_{\alpha}}\right)^{1 / 2^{*}}\left(\int_{\Omega_{\alpha} \backslash B_{R}}\left|v_{\alpha}-U\right|^{2^{*}} d v_{g_{\alpha}}\right)^{1 / 2^{* \prime}} \\
\leq & \int_{B_{R}} U\left|v_{\alpha}-U\right|^{2^{*}-1} d v_{g_{\alpha}}+C \int_{\mathbb{R}^{n} \backslash B_{R}} U^{2^{*}} d y .
\end{aligned}
$$

By taking $R$ large, the second integral can be made arbitrarily small; then, by

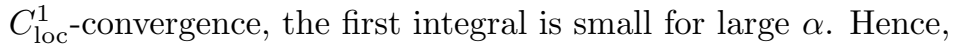

$$
\lim _{\alpha \rightarrow+\infty} \int_{\Omega_{\alpha}} U\left|v_{\alpha}-U\right|^{2^{*}-1} d v_{g_{\alpha}}=0
$$

Similarly, one easily checks that

$$
\lim _{\alpha \rightarrow+\infty} \int_{\Omega_{\alpha}} U^{2^{*}-1}\left|v_{\alpha}-U\right| d v_{g_{\alpha}}=0 .
$$

The strong convergence of the gradients is straightforward:

$$
\begin{aligned}
& \left|\int_{\Omega_{\alpha}} \nabla_{g_{\alpha}}\left(v_{\alpha}-U\right) \cdot \nabla_{g_{\alpha}} U d v_{g_{\alpha}}\right| \leq \int_{B_{R}}\left|\nabla_{g_{\alpha}}\left(v_{\alpha}-U\right)\right|\left|\nabla_{g_{\alpha}} U\right| d v_{g_{\alpha}} \\
& \quad+\left(\int_{\Omega_{\alpha} \backslash B_{R}}\left|\nabla_{g_{\alpha}}\left(v_{\alpha}-U\right)\right|^{2} d v_{g_{\alpha}}\right)^{1 / 2}\left(\int_{\Omega_{\alpha} \backslash B_{R}}\left|\nabla_{g_{\alpha}} U\right|^{2} d v_{g_{\alpha}}\right)^{1 / 2} \\
& \quad \leq \int_{B_{R}}\left|\nabla_{g_{\alpha}}\left(v_{\alpha}-U\right)\right|\left|\nabla_{g_{\alpha}} U\right| d v_{g_{\alpha}}+C\left(\int_{\Omega_{\alpha} \backslash B_{R}}|\nabla U|^{2} d y\right)^{1 / 2}
\end{aligned}
$$

and therefore

$$
\lim _{\alpha \rightarrow+\infty} \int_{\Omega_{\alpha}} \nabla_{g_{\alpha}}\left(v_{\alpha}-U\right) \cdot \nabla_{g_{\alpha}} U d v_{g_{\alpha}}=0
$$


Consequently, by (2.12) and since $\int_{\Omega_{\alpha}}\left|\nabla_{g_{\alpha}} U\right|^{2} d v_{g_{\alpha}} \rightarrow K^{-2}$, we conclude:

$$
\begin{aligned}
\int_{\Omega_{\alpha}}\left|\nabla_{g_{\alpha}}\left(v_{\alpha}-U\right)\right|^{2} d v_{g_{\alpha}}= & \int_{\Omega_{\alpha}}\left|\nabla_{g_{\alpha}} v_{\alpha}\right|^{2} d v_{g_{\alpha}}-\int_{\Omega_{\alpha}}\left|\nabla_{g_{\alpha}} U\right|^{2} d v_{g_{\alpha}} \\
& -2 \int_{\Omega_{\alpha}} \nabla_{g_{\alpha}}\left(v_{\alpha}-U\right) \cdot \nabla_{g_{\alpha}} U d v_{g_{\alpha}} \leq o(1),
\end{aligned}
$$

and (2.6) follows after a change of variables.

Corollary 2.5 (One point concentration for $u_{\alpha}$ ). For any $\varepsilon>0$ there exist $\delta_{\varepsilon}>0$ and $\alpha_{\varepsilon}>0$ such that

$$
\int_{M \backslash B_{\mu_{\alpha} / \delta_{\varepsilon}}\left(x_{\alpha}\right)}\left\{\left|\nabla_{g} u_{\alpha}\right|^{2}+u_{\alpha}^{2^{*}}\right\} d v_{g} \leq \varepsilon
$$

for all $\alpha \geq \alpha_{\varepsilon}$. In particular, for any fixed $\rho>0$,

$$
\lim _{\alpha \rightarrow+\infty} \int_{M \backslash B_{\rho}\left(x_{\alpha}\right)}\left\{\left|\nabla_{g} u_{\alpha}\right|^{2}+u_{\alpha}^{2^{*}}\right\} d v_{g}=0 .
$$

Proof. For any $\varepsilon>0$, by (2.6) and a change of variable, there exists $\delta_{\varepsilon}>0$ and $\alpha_{\varepsilon}^{\prime}$ such that for all $\alpha \geq \alpha_{\varepsilon}^{\prime}$,

$$
\int_{B_{\mu_{\alpha} / \delta_{\varepsilon}}\left(x_{\alpha}\right)}\left|\nabla_{g} u_{\alpha}\right|^{2} d v_{g} \geq \int_{\mathbb{R}^{n}}|\nabla U|^{2}-\frac{\varepsilon}{4}=K^{-2}-\frac{\varepsilon}{4},
$$

and

$$
\int_{B_{\mu_{\alpha} / \delta_{\varepsilon}}\left(x_{\alpha}\right)} u_{\alpha}^{2^{*}} d v_{g} \geq \int_{\mathbb{R}^{n}} U^{2^{*}}-\frac{\varepsilon}{4}=1-\frac{\varepsilon}{4} .
$$

Recall that $\int_{M}\left|\nabla_{g} u_{\alpha}\right|^{2} d v_{g} \rightarrow K^{-2}$ and $\int_{M} u_{\alpha}^{2^{*}} d v_{g}=1$, we can take some $\alpha_{\varepsilon} \geq \alpha_{\varepsilon}^{\prime}$ such that for all $\alpha \geq \alpha_{\varepsilon}$,

$$
\int_{M \backslash B_{\mu_{\alpha} / \delta_{\varepsilon}}\left(x_{\alpha}\right)}\left\{\left|\nabla_{g} u_{\alpha}\right|^{2}+u_{\alpha}^{2^{*}}\right\} d v_{g} \leq \varepsilon .
$$

Corollary 2.6. For any fixed $\rho>0$,

$$
\lim _{\alpha \rightarrow+\infty}\left\|u_{\alpha}\right\|_{L^{\infty}\left(M \backslash B_{\rho}\left(x_{\alpha}\right)\right)}=0 .
$$

Proof. Equation (2.2) implies the differential inequality

$$
-\Delta_{g} u_{\alpha}+\left[c(n) R_{g}-\ell_{\alpha} u_{\alpha}^{2^{*}-2}\right] u_{\alpha} \leq 0 \quad \text { on } M .
$$

By Corollary 2.5 we have, for all $x \in M \backslash B_{\rho}\left(x_{\alpha}\right)$, that $\left\|u_{\alpha}^{2^{*}-2}\right\|_{L^{n / 2}\left(B_{\rho / 2}(x)\right)}=\left\|u_{\alpha}\right\|_{L^{2^{*}}\left(B_{\rho / 2}(x)\right)}^{2^{*}-2} \leq\left\|u_{\alpha}\right\|_{L^{2^{*}}\left(M \backslash B_{\rho / 2}\left(x_{\alpha}\right)\right)}=\circ(1)$. By Moser iterations, we derive

$$
\left\|u_{\alpha}\right\|_{L^{\infty}\left(B_{\rho / 4}(x)\right)} \leq C\left\|u_{\alpha}\right\|_{L^{1}\left(B_{\rho / 2}(x)\right)} \leq C\left\|u_{\alpha}\right\|_{L^{1}(M)},
$$

and the claim follows by Proposition 2.3-(i). 
3. Uniform Estimate. The $C_{\text {loc }}^{2}\left(\mathbb{R}^{n}\right)$-convergence of the rescaled minimizer $v_{\alpha}$ to $U$ readily provides a complete description of $u_{\alpha}$ in a ball of shrinking radius $B_{\rho \mu_{\alpha}}\left(x_{\alpha}\right)$, for any $\rho>0$. In particular, it implies the estimate:

$$
u_{\alpha}(x)=\mu_{\alpha}^{-(n-2) / 2} v_{\alpha}\left(\mu_{\alpha}^{-1}\left(\exp _{x_{\alpha}} g\right)^{-1}(x)\right) \leq C(\rho) \mu_{\alpha}^{-(n-2) / 2}, \quad \forall x \in B_{\rho \mu_{\alpha}}\left(x_{\alpha}\right),
$$

and consequently

$$
u_{\alpha}(x) \leq C(\rho) \mu_{\alpha}^{(n-2) / 2} \operatorname{dist}_{g}\left(x, x_{\alpha}\right)^{2-n}, \quad \forall x \in B_{\rho \mu_{\alpha}}\left(x_{\alpha}\right) .
$$

Our aim in this section is to show that (3.1) holds uniformly on $M$. This type of estimate for minimizers has been obtained by Brezis and Peletier [13] and by Atkinson and Peletier [2] in the radially symmetric case on Euclidean balls, by Rey [37] and Han [26] on general domains in $\mathbb{R}^{n}$, and by Hebey and Vaugon [27], Li and Zhu [30] and Aubin and Li [6] on Riemannian manifolds. Our approach, similar in spirit to [30], requires new ingredients. Throughout this section, we assume $n \geq 3$.

Proposition 3.1. For every $\alpha$ sufficiently large, $u_{\alpha}$ satisfies

$$
u_{\alpha}(x) \leq C \mu_{\alpha}^{(n-2) / 2} \operatorname{dist}_{g}\left(x, x_{\alpha}\right)^{2-n}, \quad \forall x \in M .
$$

Here $C>0$ is a constant depending on $(M, g)$ only. Consequently, we have the following uniform estimate for $v_{\alpha}$ :

$$
v_{\alpha}(y) \leq \frac{C}{1+|y|^{n-2}}, \quad \forall y \in \Omega_{\alpha} .
$$

We shall prove Proposition 3.1 by showing that

$$
u_{\alpha}(x) \leq C \varphi_{\alpha}(x), \quad \forall x \in M
$$

for some $\varphi_{\alpha}>0$ satisfying:

$$
C^{-1} \mu_{\alpha}^{(n-2) / 2} \operatorname{dist}_{g}\left(x, x_{\alpha}\right)^{2-n} \leq \varphi_{\alpha}(x) \leq C \mu_{\alpha}^{(n-2) / 2} \operatorname{dist}_{g}\left(x, x_{\alpha}\right)^{2-n}, \quad \forall x \in M
$$

for every $\alpha$ sufficiently large. In fact, our main effort will be to construct a suitable such $\varphi_{\alpha}$. We set

$$
\zeta_{\alpha}=\frac{u_{\alpha}}{\varphi_{\alpha}}
$$

We have to show $\zeta_{\alpha} \leq C$ pointwise on $M$. By the conformal invariance, $\zeta_{\alpha}$ satisfies

$$
\begin{aligned}
-\Delta_{\widehat{g}_{\alpha}} \zeta_{\alpha}= & \ell_{\alpha} \zeta_{\alpha}^{2^{*}-1}-\varphi_{\alpha}^{1-2^{*}}\left(-\zeta_{\alpha} \Delta_{g} \varphi_{\alpha}+c(n) R_{g} u_{\alpha}\right. \\
& \left.+\alpha\left\|u_{\alpha}\right\|_{L^{r}(M)}^{2-r} u_{\alpha}^{r-1}\right) \quad \text { in } M \backslash\left\{x_{\alpha}\right\},
\end{aligned}
$$

where $\widehat{g}_{\alpha}$ is the metric conformal to $g$ defined in terms of $\varphi_{\alpha}$ by $\widehat{g}_{\alpha}=\varphi_{\alpha}^{4 /(n-2)} g$.

Indeed, we have

$$
-\Delta_{\widehat{g}_{\alpha}} \frac{u}{\varphi_{\alpha}}+c(n) R_{\widehat{g}_{\alpha}} \frac{u}{\varphi_{\alpha}}=\varphi_{\alpha}^{1-2^{*}}\left(-\Delta_{g} u+c(n) R_{g} u\right), \quad \forall u \in C^{2}\left(M \backslash\left\{x_{\alpha}\right\}\right) .
$$

Taking $u=\varphi_{\alpha}$, we obtain

$$
c(n) R_{\widehat{g}_{\alpha}}=\varphi_{\alpha}^{1-2^{*}}\left(-\Delta_{g} \varphi_{\alpha}+c(n) R_{g} \varphi_{\alpha}\right) .
$$

Taking $u=u_{\alpha}$, we find

$$
-\Delta_{\widehat{g}_{\alpha}} \zeta_{\alpha}=-\varphi_{\alpha}^{1-2^{*}} \Delta_{g} u_{\alpha}-c(n)\left(R_{\widehat{g}_{\alpha}}-\varphi_{\alpha}^{-4 /(n-2)} R_{g}\right) \zeta_{\alpha} .
$$


It follows that

$$
\begin{aligned}
-\Delta_{\widehat{g}_{\alpha}} \zeta_{\alpha}= & \varphi_{\alpha}^{-(n+2) /(n-2)}\left(-c(n) R_{g} u_{\alpha}-\alpha\left\|u_{\alpha}\right\|_{L^{r}(M)}^{2-r} u_{\alpha}^{r-1}+\ell_{\alpha} u_{\alpha}^{2^{*}-1}\right) \\
& +\varphi_{\alpha}^{1-2^{*}} \zeta_{\alpha} \Delta_{g} \varphi_{\alpha},
\end{aligned}
$$

which implies (3.6).

By the uniform estimate (3.5), the metrics $\widehat{g}_{\alpha}$ satisfy a Sobolev inequality with a constant independent of $\alpha$ :

Lemma 3.2. There exists a constant $C>0$ independent of $\alpha$ such that for all $u \in H^{1}(M), u \equiv 0$ in a neighborhood of $x_{\alpha}$ :

$$
\left(\int_{M}|u|^{2^{*}} d v_{\widehat{g}_{\alpha}}\right)^{2 / 2^{*}} \leq C \int_{M}\left|\nabla_{\widehat{g}_{\alpha}} u\right|^{2} d v_{\widehat{g}_{\alpha}} .
$$

Proof. It is well-known (see, e.g., Appendix A in [30]) that there exists a constant $C=C(M, g)$ such that for all $x_{0} \in M, u \in H^{1}(M), u \equiv 0$ in a neighborhood of $x_{0}$, there holds:

$$
\left(\int_{M} \frac{|u|^{2^{*}}}{\operatorname{dist}_{g}\left(x, x_{0}\right)^{2 n}} d v_{g}\right)^{2 / 2^{*}} \leq C \int_{M} \frac{\left|\nabla_{g} u\right|^{2}}{\operatorname{dist}_{g}\left(x, x_{0}\right)^{2 n-4}} d v_{g} .
$$

Now it suffices to observe that by conformality of $\widehat{g}_{\alpha}$ we have:

$$
d v_{\widehat{g}_{\alpha}}=\varphi_{\alpha}^{2^{*}} d v_{g} \quad \text { and } \quad\left|\nabla_{\widehat{g}_{\alpha}} u\right|^{2}=\varphi_{\alpha}^{-4 /(n-2)}\left|\nabla_{g} u\right|^{2},
$$

and to recall (3.5).

At this point it is clear from (3.6) that if we can find a function $\varphi_{\alpha}>0$ satisfying (3.5) and such that:

$$
-\frac{u_{\alpha}}{\varphi_{\alpha}} \Delta_{g} \varphi_{\alpha}+c(n) R_{g} u_{\alpha}+\alpha\left\|u_{\alpha}\right\|_{L^{r}(M)}^{2-r} u_{\alpha}^{r-1} \geq 0 \quad \text { in } M \backslash\left\{x_{\alpha}\right\},
$$

then the corresponding $\zeta_{\alpha}$ will satisfy:

$$
\begin{aligned}
& -\Delta_{\widehat{g}_{\alpha}} \zeta_{\alpha} \leq \ell_{\alpha} \zeta_{\alpha}^{2^{*}-1} \quad \text { in } M \backslash\left\{x_{\alpha}\right\} \\
& \int_{M \backslash B_{\mu_{\alpha} / \delta_{1}}\left(x_{\alpha}\right)} \zeta_{\alpha}^{2^{*}} d v_{\widehat{g}_{\alpha}}=\int_{M \backslash B_{\mu_{\alpha} / \delta_{1}}\left(x_{\alpha}\right)} u_{\alpha}^{2^{*}} d v_{g} \leq \varepsilon,
\end{aligned}
$$

(recall Corollary 2.5 in Section 2). For any $\rho>0$, let

$$
R_{i}:=\frac{\left[2-2^{-(i-1)}\right] \mu_{\alpha}}{\rho}, \quad i=1,2,3, \ldots
$$

By (3.5) we may choose cutoff functions $\eta_{i}$ (depending on $\alpha$ ) satisfying:

$$
\begin{aligned}
& \eta_{i} \equiv 1 \quad \text { in } M \backslash B_{R_{i+1}} \\
& \eta_{i} \equiv 0 \quad \text { in } M \backslash B_{R_{i}} \\
& \left|\nabla_{\widehat{g}_{\alpha}} \eta_{i}\right| \leq C(\rho) 2^{i}, \quad\left|\nabla_{\widehat{g}_{\alpha}}^{2} \eta_{i}\right| \leq C(\rho) 4^{i} .
\end{aligned}
$$

Then we shall have all necessary ingredients to apply the Moser iteration technique to (3.10) and to derive:

LEMMA 3.3. The following pointwise upper bound holds:

$$
\zeta_{\alpha} \leq C \quad \text { in } M \backslash B_{\mu_{\alpha} / \delta_{0}}\left(x_{\alpha}\right) .
$$

Proof. By applying Moser iterations to (3.10), see [30] for the detailed proof. 
Estimates (3.1) and (3.11) will then imply (3.4) and thus Proposition 3.1 will be established.

We note that (3.9) is trivially satisfied if $u_{\alpha}=0$. In $\left(M \backslash\left\{x_{\alpha}\right\}\right) \cap\left\{u_{\alpha}>0\right\},(3.9)$ is equivalent to:

$$
-\Delta_{g} \varphi_{\alpha}+\left[c(n) R_{g}+\alpha\left(\frac{\left\|u_{\alpha}\right\|_{L^{r}(M)}}{u_{\alpha}}\right)^{2-r}\right] \varphi_{\alpha} \geq 0,
$$

and the operator on the left hand side above is linear in $\varphi_{\alpha}$. Furthermore, the blowup rate as in (3.5) is satisfied if $\mu_{\alpha}^{(2-n) / 2} \varphi_{\alpha}$ has the blowup rate of the Green's function with pole at $x_{\alpha}$. In fact, we shall obtain a $\varphi_{\alpha}$ of the form $\varphi_{\alpha}=\mu_{\alpha}^{(n-2) / 2} G_{\alpha}$, with $G_{\alpha}$ the Green's function for the operator $-\Delta_{g}+\widetilde{V}_{\alpha}$ with pole at $x_{\alpha}$, and where $\widetilde{V}_{\alpha}$ is a truncation of the "potential" $c(n) R_{g}+\alpha\left(\left\|u_{\alpha}\right\|_{L^{r}(M)} / u_{\alpha}\right)^{2-r}$ appearing in (3.12). The detailed proof follows.

We define a function $\widetilde{V}_{\alpha}$ in the following way:

$$
\widetilde{V}_{\alpha}:= \begin{cases}\min \left\{c(n) R_{g}+\alpha\left(\frac{\left\|u_{\alpha}\right\|_{L^{r}(M)}}{u_{\alpha}}\right)^{2-r}, 1\right\}, & \text { if } u_{\alpha} \neq 0, \\ 1, & \text { if } u_{\alpha}=0 .\end{cases}
$$

Note that $\widetilde{V}_{\alpha}$ is Lipschitz on $M$ (with Lipschitz constant depending on $\alpha$ ) and it is uniformly bounded:

$$
-c(n)\left\|R_{g}\right\|_{\infty} \leq \widetilde{V}_{\alpha} \leq 1 .
$$

We shall prove (3.4) with $\varphi_{\alpha}=\mu_{\alpha}^{(n-2) / 2} G_{\alpha}$ and $G_{\alpha}$ defined in the following

Proposition 3.4. The operators $-\Delta_{g}+\widetilde{V}_{\alpha}$ are coercive on $H^{1}(M)$ for sufficiently large $\alpha$, with coercivity constant uniform in $\alpha$. Consequently, for every $\alpha$ sufficiently large there exists a unique (distributional) solution $G_{\alpha}$ to the equation:

$$
-\Delta_{g} G_{\alpha}+\widetilde{V}_{\alpha} G_{\alpha}=\delta_{x_{\alpha}}, \quad \text { on } M
$$

Furthermore, the first nonzero eigenvalue of $-\Delta_{g}+\widetilde{V}_{\alpha}$ is bounded away from zero and therefore $G_{\alpha}$ satisfies, for some constant $C>0$ independent of $\alpha$,

(i) $G_{\alpha} \in C_{l o c}^{2}\left(M \backslash\left\{x_{\alpha}\right\}\right)$;

(ii) $C^{-1} \operatorname{dist}_{g}\left(x, x_{\alpha}\right)^{2-n} \leq G_{\alpha}(x) \leq C \operatorname{dist}_{g}\left(x, x_{\alpha}\right)^{2-n} \quad \forall x \in M$;

(iii) $u_{\alpha} \Delta_{g} G_{\alpha} \leq\left[c(n) R_{g} u_{\alpha}+\alpha\left\|u_{\alpha}\right\|_{r}^{2-r} u_{\alpha}^{r-1}\right] G_{\alpha}$ in $M \backslash\left\{x_{\alpha}\right\}$.

In order to prove Proposition 3.4 we need the following

Lemma 3.5. The functions $\widetilde{V}_{\alpha}$ satisfy:

$$
\lim _{\alpha \rightarrow+\infty} \operatorname{vol}_{g}\left\{\widetilde{V}_{\alpha}<\frac{1}{2}\right\}=0 .
$$

Proof. Note that for every measurable set $E$ such that $\bar{E} \subset M \cap\left\{u_{\alpha}>0\right\}$ we have the lower bound:

$$
\left\|u_{\alpha}\right\|_{L^{r}(E)}\left\|u_{\alpha}^{-1}\right\|_{L^{r}(E)} \geq\left(\operatorname{vol}_{g} E\right)^{2 / r}
$$

Indeed, using the Hölder inequality we find:

$$
\operatorname{vol}_{g} E=\int_{E} d v_{g}=\int_{E} u_{\alpha}^{r / 2} u_{\alpha}^{-r / 2} d v_{g} \leq\left\|u_{\alpha}\right\|_{L^{r}(E)}^{r / 2}\left\|u_{\alpha}^{-1}\right\|_{L^{r}(E)}^{r / 2} .
$$


It follows that

$$
\begin{aligned}
\left\|\left(\left\|u_{\alpha}\right\|_{L^{r}(M)} u_{\alpha}^{-1}\right)^{2-r}\right\|_{L^{r /(2-r)}(E)} & =\left\|u_{\alpha}\right\|_{L^{r}(M)}^{2-r}\left\|u_{\alpha}^{-(2-r)}\right\|_{L^{r /(2-r)}(E)} \\
& \geq\left\|u_{\alpha}\right\|_{L^{r}(E)}^{2-r}\left\|u_{\alpha}^{-1}\right\|_{L^{r}(E)}^{2-r} \geq|E|^{(2-r) 2 / r} .
\end{aligned}
$$

Let $E_{\alpha}:=\left\{\widetilde{V}_{\alpha}<1 / 2\right\}$. Then $\bar{E}_{\alpha} \subset M \cap\left\{u_{\alpha}>0\right\}$ and therefore, by (3.15),

$$
\left(\operatorname{vol}_{g} E_{\alpha}\right)^{(2-r) 2 / r} \leq\left\|\left(\left\|u_{\alpha}\right\|_{L^{r}(M)} u_{\alpha}^{-1}\right)^{2-r}\right\|_{L^{r /(2-r)}\left(E_{\alpha}\right)} .
$$

On the other hand, since

$$
\alpha\left(\left\|u_{\alpha}\right\|_{L^{r}(M)} u_{\alpha}^{-1}\right)^{2-r}<\frac{1}{2}+c(n)\left|R_{g}\right|, \quad \text { on } E_{\alpha},
$$

we have

$$
\alpha\left\|\left(\left\|u_{\alpha}\right\|_{L^{r}(M)} u_{\alpha}^{-1}\right)^{2-r}\right\|_{L^{r /(2-r)}\left(E_{\alpha}\right)} \leq\left(\frac{1}{2}+c(n)\left\|R_{g}\right\|_{L^{\infty}(M)}\right)\left(\operatorname{vol}_{g} M\right)^{(2-r) / r},
$$

and consequently,

$$
\alpha\left(\operatorname{vol}_{g} E_{\alpha}\right)^{(2-r) / r} \leq C,
$$

for some $C>0$ independent of $\alpha$. Now Lemma 3.5 follows immediately.

Proof of Proposition 3.4. Proof of the coercivity. For $\tilde{\gamma}=1 / 2$ and $u \in H^{1}(M)$, by the Sobolev inequality and a straightforward computation we have:

$$
\begin{aligned}
& \int_{M}\left\{\left|\nabla_{g} u\right|^{2}+\widetilde{V}_{\alpha} u^{2}\right\} d v_{g}=\int_{M}\left\{\left|\nabla_{g} u\right|^{2}+\tilde{\gamma} u^{2}+\left(\widetilde{V}_{\alpha}-\tilde{\gamma}\right) u^{2}\right\} d v_{g} \\
& \quad \geq \int_{M}\left\{\left|\nabla_{g} u\right|^{2}+\tilde{\gamma} u^{2}-\left(\widetilde{V}_{\alpha}-\tilde{\gamma}\right)_{-} u^{2}\right\} d v_{g} \\
& \geq \int_{M}\left\{\left|\nabla_{g} u\right|^{2}+\tilde{\gamma} u^{2}\right\} d v_{g}-\left\|\left(\widetilde{V}_{\alpha}-\tilde{\gamma}\right)_{-}\right\|_{L^{n / 2}(M)}\|u\|_{L^{2^{*}}(M)}^{2} \\
& \geq \int_{M}\left\{\left|\nabla_{g} u\right|^{2}+\tilde{\gamma} u^{2}\right\} d v_{g}-C \operatorname{vol}_{g}\left\{\widetilde{V}_{\alpha}<1 / 2\right\} \int_{M}\left\{\left|\nabla_{g} u\right|^{2}+u^{2}\right\} d v_{g},
\end{aligned}
$$

where $\left(\widetilde{V}_{\alpha}-\tilde{\gamma}\right)_{-} \geq 0$ denotes the negative part of $\widetilde{V}_{\alpha}-\tilde{\gamma}$. The coercivity and its uniformity in $\alpha$ follow from the above and Lemma 3.5.

Proof of (i) and (ii). Because of the coercivity of $-\Delta_{g}+\widetilde{V}_{\alpha}$, the Lipschitz regularity and the uniform $L^{\infty}$ bound of $\widetilde{V}_{\alpha}$, it follows from standard elliptic theories (see e.g., [24], [41] and [21]) that $G_{\alpha}$ is uniquely defined by (3.14) and it satisfies (i) and (ii).

Proof of (iii). Since $G_{\alpha} \in C_{\text {loc }}^{2}\left(M \backslash\left\{x_{\alpha}\right\}\right)$ we only need to check the inequality pointwise. If $u_{\alpha}=0$ it is trivial. So assume $u_{\alpha}>0$. By (3.14) we have

$$
-\Delta_{g} G_{\alpha}+\widetilde{V}_{\alpha} G_{\alpha}=0 \quad \text { pointwise in } M \backslash\left\{x_{\alpha}\right\} .
$$

Since $G_{\alpha}>0$, using the definition of $\widetilde{V}_{\alpha}$, we have

$$
\Delta_{g} G_{\alpha}=\widetilde{V}_{\alpha} G_{\alpha} \leq\left[c(n) R_{g}+\alpha\left(\frac{\left\|u_{\alpha}\right\|_{L^{r}(M)}}{u_{\alpha}}\right)^{2-r}\right] G_{\alpha},
$$

pointwise in $\left(M \backslash\left\{x_{\alpha}\right\}\right) \cap\left\{u_{\alpha}>0\right\}$. Multiplying the inequality above by $u_{\alpha}$, we again obtain (iii). Proposition 3.4 is established.

Proof of Proposition 3.1. The estimate for $u_{\alpha}$ follows by (3.1) and Lemma 3.3. Since $v_{\alpha}$ is uniformly bounded in $|y|<1,(3.3)$ follows from the estimate of $u_{\alpha}$ by a change of variables. 
4. Energy Estimate. We shall need estimates for the convergence rates of the limits "in energy" obtained in Section 2. The pointwise estimates obtained in Section 2 allow us to adapt the energy estimates of Bahri-Coron [7].

In order to simplify calculations, we introduce a conformal metric $\widehat{g}=\psi^{4 /(n-2)} g$, with $\psi \in C^{\infty}(M), \psi\left(x_{\alpha}\right)=1, \frac{1}{2} \leq \psi \leq 2,\|\psi\|_{C^{2}} \leq C$, such that $R_{\widehat{g}} \equiv 0$ in $B_{\delta_{0}}(P)$, and where $\delta_{0}$ is a suitably chosen small constant and both $\delta_{0}$ and $C$ depend only on $(M, g)$. Such a metric may be obtained by locally solving

$$
-\Delta_{g} \psi+c(n) R_{g} \psi=0 \quad \text { in } B_{\delta_{0}}
$$

and then extending $\psi$ smoothly to $M$. We denote, for $\delta_{0} / 2 \leq \delta_{\alpha} \leq \delta_{0}$,

$$
B_{\alpha}=B_{\delta_{\alpha}}^{\widehat{g}}\left(x_{\alpha}\right) \text {. }
$$

For $\tilde{x} \in B_{\alpha}$ and $\lambda>0$, we consider

$$
\xi_{\tilde{x}, \lambda}^{\widehat{g}}(x)=\left(\frac{\lambda}{1+(\lambda \bar{\lambda})^{2} \operatorname{dist}_{\tilde{g}}^{2}(x, \tilde{x})^{2}}\right)^{\frac{n-2}{2}}, \quad \forall x \in B_{\alpha} .
$$

It follows from Proposition 2.4 that

$$
\lim _{\alpha \rightarrow+\infty} \int_{B_{\alpha}}\left\{\left|\nabla_{\widehat{g}}\left(\frac{u_{\alpha}}{\psi}-\xi_{x_{\alpha}, \mu_{\alpha}^{-1}}^{\widehat{g}}\right)\right|^{2}+\left|\frac{u_{\alpha}}{\psi}-\xi_{x_{\alpha}, \mu_{\alpha}^{-1}}^{\widehat{g}}\right|^{2^{*}}\right\} d v_{\widehat{g}}=0 .
$$

We follow the idea in [7] of selecting for every $\alpha$ an optimal multiple of a $\widehat{g}$ bubble, denoted $t_{\alpha} \xi_{\alpha}=t_{\alpha} \xi_{\tilde{x}_{\alpha}, \lambda_{\alpha}}^{\widehat{g}}$, and of estimating the difference $u_{\alpha} / \psi-t_{\alpha} \xi_{\alpha}$ by exploiting orthogonality. For future convenience, we prove our estimates for $n \geq 3$. For $\tilde{x} \in B_{\mu_{\alpha} \delta_{\alpha} / 2}^{\hat{g}}\left(x_{\alpha}\right)$ and $\lambda>0$, let $h_{\tilde{x}, \lambda}$ be defined by:

$$
\begin{cases}\Delta_{\widetilde{g}} h_{\tilde{x}, \lambda}=0 & \text { in } B_{\alpha}, \\ h_{\tilde{x}, \lambda}=\xi_{\tilde{x}, \lambda}^{\widehat{g}} & \text { on } \partial B_{\alpha},\end{cases}
$$

and let $\chi_{\alpha}$ be defined by

$$
\begin{cases}\Delta_{\widehat{g}} \chi_{\alpha}=0 & \text { in } B_{\alpha}, \\ \chi_{\alpha}=\frac{u_{\alpha}}{\psi} & \text { on } \partial B_{\alpha} .\end{cases}
$$

Then $u_{\alpha} / \psi-\chi_{\alpha} \in H_{0}^{1}\left(B_{\alpha}\right), \xi_{\tilde{x}, \lambda}^{\widehat{g}}-h_{\tilde{x}, \lambda} \in H_{0}^{1}\left(B_{\alpha}\right)$ are the projections of $u_{\alpha}$ and $\xi_{\tilde{x}, \lambda}^{\widehat{g}}$, respectively, on $H_{0}^{1}\left(B_{\alpha}\right)$. We set

$$
\sigma_{\tilde{x}, \lambda}=\xi_{\tilde{x}, \lambda}^{\widehat{g}}-h_{\tilde{x}, \lambda} .
$$

Then $\sigma_{\tilde{x}, \lambda} \leq \xi_{\tilde{x}, \lambda}^{\widehat{g}}$ satisfies:

$$
\begin{cases}\Delta_{\widetilde{g}} \sigma_{\tilde{x}, \lambda}=\Delta_{\widehat{g}} \xi_{\tilde{x}, \lambda}^{\widehat{g}} & \text { in } B_{\alpha} \\ \sigma_{\tilde{x}, \lambda}=0 & \text { on } \partial B_{\alpha} .\end{cases}
$$

Let $\left(t_{\alpha}, \tilde{x}_{\alpha}, \lambda_{\alpha}\right) \in\left[\frac{1}{2}, \frac{3}{2}\right] \times \overline{B_{\mu_{\alpha} \delta_{\alpha} / 2}^{\hat{g}}\left(x_{\alpha}\right)} \times\left[\frac{1}{2 \mu_{\alpha}}, \frac{3}{2 \mu_{\alpha}}\right]$ be such that

$$
\begin{aligned}
& \left\|\frac{u_{\alpha}}{\psi}-\chi_{\alpha}-t_{\alpha} \sigma_{\tilde{x}_{\alpha}, \lambda_{\alpha}}\right\|_{\widehat{g}} \\
& =\min \left\{\left\|\frac{u_{\alpha}}{\psi}-\chi_{\alpha}-t \sigma_{\tilde{x}, \lambda}\right\|_{\hat{g}}: \begin{array}{c}
|t-1| \leq 1 / 2, \tilde{x} \in \overline{B_{\mu_{\alpha} \delta_{\alpha} / 2}^{\hat{g}}\left(x_{\alpha}\right)} \\
\left|\mu_{\alpha} \lambda-1\right| \leq 1 / 2
\end{array}\right\} .
\end{aligned}
$$


To simplify notation, henceforth we denote:

$$
\sigma_{\alpha}=\sigma_{\tilde{x}_{\alpha}, \lambda_{\alpha}}, \quad \xi_{\alpha}=\xi_{\tilde{x}_{\alpha}, \lambda_{\alpha}}^{\widehat{\widehat{g}}}, \quad h_{\alpha}=h_{\tilde{x}_{\alpha}, \lambda_{\alpha}},
$$

and we set:

$$
w_{\alpha}=\frac{u_{\alpha}}{\psi}-\chi_{\alpha}-t_{\alpha} \sigma_{\alpha} .
$$

The main result in this section is the following estimate for $w_{\alpha}$ :

Proposition 4.1 (Energy estimate). For $n \geq 3$, we have:

$$
\begin{aligned}
& \left\|w_{\alpha}\right\|+\left|t_{\alpha}^{2^{*}-2} \ell_{\alpha}-K^{-2}\right| \\
\leq & C\left(\mu_{\alpha}^{2}\|U\|_{2^{* \prime}, \mu_{\alpha}^{-1}}+\varepsilon_{\alpha}\left\|U^{r-1}\right\|_{2^{* \prime}, \mu_{\alpha}^{-1}}+\mu_{\alpha}^{n-2}\left\|U^{2^{*}-2}\right\|_{2^{* \prime}, \mu_{\alpha}^{-1}} .\right.
\end{aligned}
$$

Recall from Section 2 that $\varepsilon_{\alpha}=\mu_{\alpha}^{n-\frac{n-2}{2} r} \alpha\left\|u_{\alpha}\right\|_{L^{r}(M)}^{2-r}=\circ_{\alpha}(1)$. We define

$$
W_{\alpha}=\left\{w \in H_{0}^{1}\left(B_{\alpha}\right): \quad \begin{array}{c}
\left\langle\sigma_{\alpha}, w\right\rangle_{\widehat{g}}=0 \\
\langle f, w\rangle_{\widehat{g}}=0 \quad \forall f \in E
\end{array}\right\},
$$

where $E \subset H_{0}^{1}\left(B_{\alpha}\right)$ is the tangent space at $\sigma_{\tilde{x}_{\alpha}, \lambda_{\alpha}}$ of the finite dimensional surface $\left\{\sigma_{\tilde{x}, \lambda}: \tilde{x} \in B_{\mu_{\alpha} \delta_{\alpha}}\left(x_{\alpha}\right), \lambda>0\right\} \subset H_{0}^{1}\left(B_{\alpha}\right)$, with respect to the metric induced by the inner product $\langle u, v\rangle_{\widehat{g}}=\int_{B_{\alpha}} \nabla_{\widehat{g}} u \cdot \nabla_{\widehat{g}} v d v_{\widehat{g}}$. We work with coordinates given by the exponential map $\exp _{\tilde{x}_{\alpha}}(y), y=\left(y^{i}\right), i=1, \ldots, n$, we can write

$$
E=\operatorname{span}\left\{\frac{\partial \sigma_{\alpha}}{\partial y^{i}}, i=1, \ldots, n, \frac{\partial \sigma_{\alpha}}{\partial \lambda}\right\}
$$

where

$$
\frac{\partial \sigma_{\alpha}}{\partial y^{i}}=\left.\frac{\partial \sigma_{\exp _{\tilde{x}_{\alpha}}(y), \lambda_{\alpha}}}{\partial y^{i}}\right|_{y=0}, \quad \frac{\partial \sigma_{\alpha}}{\partial \lambda}=\left.\frac{\partial \sigma_{\tilde{x}_{\alpha}, \lambda}}{\partial \lambda}\right|_{\lambda=\lambda_{\alpha}} .
$$

Lemma 4.2. For some constant $C$ independent of $\alpha$,

$$
\int_{B_{\alpha}}\left|\nabla_{\widehat{g}} h_{\alpha}\right|^{2} d v_{\widehat{g}} \leq C \mu_{\alpha}^{n-2} .
$$

Proof. By standard elliptic estimates and properties of $\xi_{\alpha}$, we have that

$$
\int_{B_{\alpha}}\left|\nabla_{\widehat{g}} h_{\alpha}\right|^{2} d v_{\widehat{g}} \leq C\left(\int_{\partial B_{\alpha}}\left|\nabla_{\widehat{g}} \xi_{\alpha}\right|^{2} d s_{\widehat{g}}+\int_{\partial B_{\alpha}} \xi_{\alpha}^{2} d s_{\widehat{g}}\right) \leq C \mu_{\alpha}^{n-2} .
$$

We observe that by the uniform estimate (3.2) and by the maximum principle,

$$
\left\|h_{\alpha}\right\|_{L^{\infty}\left(B_{\alpha}\right)}+\left\|\chi_{\alpha}\right\|_{L^{\infty}\left(B_{\alpha}\right)} \leq C \mu_{\alpha}^{(n-2) / 2} .
$$

It follows that $\left|\sigma_{\alpha}\right| \leq C \xi_{\alpha}$ on $B_{\alpha}$. Using Proposition 2.4, it is not difficult to see that:

Lemma 4.3. As $\alpha \rightarrow+\infty$, we have $\left\|w_{\alpha}\right\| \rightarrow 0, t_{\alpha} \rightarrow 1, \mu_{\alpha}^{-1} \operatorname{dist}_{\hat{g}}\left(x_{\alpha}, \tilde{x}_{\alpha}\right) \rightarrow 0$, $\mu_{\alpha} \lambda_{\alpha} \rightarrow 1$. Furthermore, $w_{\alpha} \in W_{\alpha}$.

Proof. By definition of $t_{\alpha}$ and $\sigma_{\alpha}$,

$$
\begin{aligned}
\left\|t_{\alpha} \sigma_{\alpha}-\sigma_{x_{\alpha}, \mu_{\alpha}^{-1}}\right\| & \leq\left\|\frac{u_{\alpha}}{\psi}-\chi_{\alpha}-t_{\alpha} \sigma_{\alpha}\right\|+\left\|\frac{u_{\alpha}}{\psi}-\chi_{\alpha}-\sigma_{x_{\alpha}, \mu_{\alpha}^{-1}}\right\| \\
& \leq 2\left\|\frac{u_{\alpha}}{\psi}-\chi_{\alpha}-\sigma_{x_{\alpha}, \mu_{\alpha}^{-1}}\right\| \leq 2\left\|\frac{u_{\alpha}}{\psi}-\xi_{x_{\alpha}, \mu_{\alpha}^{-1}}^{\hat{g}}\right\| .
\end{aligned}
$$


In the last step we have used $\Delta_{\widehat{g}}\left(\frac{u_{\alpha}}{\psi}-\xi_{x_{\alpha}, \mu_{\alpha}^{-1}}^{\hat{g}}\right)=\Delta_{\widehat{g}}\left(\frac{u_{\alpha}}{\psi}-\chi_{\alpha}-\sigma_{x_{\alpha}, \mu_{\alpha}^{-1}}\right)$ in $B_{\alpha}$ and $\frac{u_{\alpha}}{\psi}-\chi_{\alpha}-\sigma_{x_{\alpha}, \mu_{\alpha}^{-1}}=0$ on $\partial B_{\alpha}$. Hence, in view of (4.1), $\left\|t_{\alpha} \sigma_{\alpha}-\sigma_{x_{\alpha}, \mu_{\alpha}^{-1}}\right\| \rightarrow 0$ and $\left\|w_{\alpha}\right\| \rightarrow 0$. By the arguments in Lemma 4.2, we have $\left\|h_{\tilde{x}, \lambda}\right\| \leq C \lambda^{-(n-2) / 2}$ if $\operatorname{dist}_{\tilde{g}}\left(\tilde{x}, x_{\alpha}\right) \leq \delta_{\alpha} / 2$. Consequently, we derive

$$
\left\|t_{\alpha} \xi_{\alpha}-\xi_{x_{\alpha}, \mu_{\alpha}^{-1}}^{\hat{g}}\right\| \leq\left\|t_{\alpha} \sigma_{\alpha}-\sigma_{x_{\alpha}, \mu_{\alpha}^{-1}}\right\|+\left\|t_{\alpha} h_{\alpha}\right\|+\left\|h_{x_{\alpha}, \mu_{\alpha}^{-1}}\right\| \rightarrow 0
$$

as $\alpha \rightarrow+\infty$. It follows that $t_{\alpha} \rightarrow 1, \mu_{\alpha}^{-1} \operatorname{dist}_{\hat{g}}\left(x_{\alpha}, \tilde{x}_{\alpha}\right) \rightarrow 0$, and $\mu_{\alpha} \lambda_{\alpha} \rightarrow 1$. Therefore the minimum of the norm is attained in the interior of $\left[\frac{1}{2}, \frac{3}{2}\right] \times \overline{B_{\mu_{\alpha} \delta_{\alpha} / 2}\left(x_{\alpha}\right)} \times$ $\left[\frac{1}{2 \mu_{\alpha}}, \frac{3}{2 \mu_{\alpha}}\right]$. Now a straightforward variational argument yields $w_{\alpha} \in W_{\alpha}$.

In order to estimate $w_{\alpha}$, we begin by writing an equation for $w_{\alpha}$ :

LEMMA 4.4. $w_{\alpha}$ satisfies:

$$
-\Delta_{\widehat{g}} w_{\alpha}-k_{\alpha}\left|\Theta_{\alpha}\right|^{2^{*}-3} \Theta_{\alpha} w_{\alpha}+b^{\prime}\left|\Theta_{\alpha}\right|^{2^{*}-3} w_{\alpha}^{2}+b^{\prime \prime}\left|w_{\alpha}\right|^{2^{*}-1}=f_{\alpha} \quad \text { in } B_{\alpha},
$$

where

$$
\begin{aligned}
& k_{\alpha}=\left(2^{*}-1\right) \ell_{\alpha}, \\
& \Theta_{\alpha}=t_{\alpha} \sigma_{\alpha}+\chi_{\alpha}, \\
& f_{\alpha}=\ell_{\alpha}\left(t_{\alpha} \xi_{\alpha}\right)^{2^{*}-1}+t_{\alpha} \Delta_{\widehat{g}} \xi_{\alpha}-\alpha\left\|u_{\alpha}\right\|_{L^{r}(M)}^{2-r} \psi^{1-2^{*}} u_{\alpha}^{r-1}+O\left(\mu_{\alpha}^{(n-2) / 2} \xi_{\alpha}^{2^{*}-2}\right),
\end{aligned}
$$

and where $b^{\prime}, b^{\prime \prime}$ are bounded functions with $b^{\prime} \equiv 0$ if $n \geq 6$.

Proof. From (2.2), using the conformal invariance (1.8) and recalling that $R_{\widehat{g}} \equiv 0$ in $B_{\alpha}$, we have that $u_{\alpha} / \psi$ satisfies:

$$
-\Delta_{\widehat{g}} \frac{u_{\alpha}}{\psi}+\alpha\left\|u_{\alpha}\right\|_{L^{r}(M)}^{2-r} \psi^{1-2^{*}} u_{\alpha}^{r-1}=\ell_{\alpha}\left(\frac{u_{\alpha}}{\psi}\right)^{2^{*}-1} \quad \text { in } B_{\alpha} .
$$

Consequently, $w_{\alpha}$ satisfies:

$$
-\Delta_{\widehat{g}} w_{\alpha}=\ell_{\alpha}\left(\Theta_{\alpha}+w_{\alpha}\right)^{2^{*}-1}+t_{\alpha} \Delta_{\widehat{g}} \sigma_{\alpha}-\alpha\left\|u_{\alpha}\right\|_{L^{r}(M)}^{2-r} \psi^{1-2^{*}} u_{\alpha}^{r-1} \quad \text { in } B_{\alpha} .
$$

In order to simplify the right hand side in (4.6), we use the elementary expansion:

$$
\begin{aligned}
(x+y)^{2^{*}-1}= & |x|^{2^{*}-2} x+\left(2^{*}-1\right)|x|^{2^{*}-3} x y \\
& +b^{\prime}(x, y)|x|^{2^{*}-3} y^{2}+b^{\prime \prime}(x, y)|y|^{2^{*}-1},
\end{aligned}
$$

for all $x, y \in \mathbb{R}$ such that $x+y \geq 0$, where $b^{\prime}, b^{\prime \prime}$ are bounded functions and $b^{\prime} \equiv 0$ if $n \geq 6$. For $x=\Theta_{\alpha}$ and $y=w_{\alpha}$, we obtain:

$$
\begin{aligned}
\left(\Theta_{\alpha}+w_{\alpha}\right)^{2^{*}-1}= & \left|\Theta_{\alpha}\right|^{2^{*}-2} \Theta_{\alpha}+\left(2^{*}-1\right)\left|\Theta_{\alpha}\right|^{2^{*}-3} \Theta_{\alpha} w_{\alpha} \\
& +b^{\prime}\left|\Theta_{\alpha}\right|^{2^{*}-3} w_{\alpha}^{2}+b^{\prime \prime}\left|w_{\alpha}\right|^{2^{*}-1} .
\end{aligned}
$$

Note that $\Theta_{\alpha}=t_{\alpha} \xi_{\alpha}-t_{\alpha} h_{\alpha}+\chi_{\alpha}$. By (4.4) and properties of $\xi_{\alpha}$, we have $\mid \chi_{\alpha}-$ $t_{\alpha} h_{\alpha} \mid \leq C \mu_{\alpha}^{(n-2) / 2} \leq C t_{\alpha} \xi_{\alpha}$, and thus by simple calculus:

$$
\begin{aligned}
\left|\Theta_{\alpha}\right|^{2^{*}-2} \Theta_{\alpha} & =\left|t_{\alpha} \xi_{\alpha}-t_{\alpha} h_{\alpha}+\chi_{\alpha}\right|^{2^{*}-2}\left(t_{\alpha} \xi_{\alpha}-t_{\alpha} h_{\alpha}+\chi_{\alpha}\right) \\
& =\left(t_{\alpha} \xi_{\alpha}\right)^{2^{*}-1}+O\left(\mu_{\alpha}^{(n-2) / 2} \xi_{\alpha}^{2^{*}-2}\right) .
\end{aligned}
$$

Inserting the above expansions into (4.6), we obtain (4.5). 
The proof of Proposition 4.1 relies on the coercivity property as in Lemma 4.5 below. Recall that $\delta_{0}>0$ was introduced in Section 2 as an upper bound for the radii $\delta_{\alpha}$ of the balls $B_{\alpha}=B_{\delta_{\alpha}}\left(x_{\alpha}\right)$. Here is where we fix $\delta_{0}$. We denote by $Q_{\alpha}$ the continuous bilinear form defined for $\varphi, \psi \in H_{0}^{1}\left(B_{\alpha}\right)$ by:

$$
Q_{\alpha}(\varphi, \psi)=\int_{B_{\alpha}}\left\{\nabla_{\widehat{g}} \varphi \cdot \nabla_{\widehat{g}} \psi-k_{\alpha}\left|\Theta_{\alpha}\right|^{2^{*}-3} \Theta_{\alpha} \varphi \psi\right\} d v_{\widehat{g}}
$$

where $k_{\alpha}$ and $\Theta_{\alpha}$ are defined in Lemma 4.4.

LEMmA 4.5. There exist $0<\delta_{0} \ll 1, \alpha_{0} \gg 1$ and $c_{0}>0$ independent of $\alpha \geq \alpha_{0}$ such that

$$
Q_{\alpha}(w, w) \geq c_{0} \int_{B_{\alpha}}\left|\nabla_{\widehat{g}} w\right|^{2} d v_{\widehat{g}}, \quad \forall w \in W_{\alpha}, \forall \alpha \geq \alpha_{0} .
$$

Lemma 4.5 is a consequence of the following general perturbation result:

Lemma 4.6. Let $\Omega \subset \mathbb{R}^{n}$, let $h$ be a metric on $\Omega, k>0$ and $\Theta \in L^{2^{*}}(\Omega)$. Denote by $Q$ the continuous bilinear form defined on $H_{0}^{1}(\Omega) \times H_{0}^{1}(\Omega)$ by

$$
Q(\varphi, \psi)=\int_{\Omega}\left\{\nabla_{h} \varphi \cdot \nabla_{h} \psi-k|\Theta|^{2^{*}-3} \Theta \varphi \psi\right\} d v_{h}
$$

There exist $\varepsilon_{0}>0$ and $c_{1}>0$, depending only on $n$, such that if

$$
\|\Theta-U\|_{L^{2^{*}(\Omega)}}+\left|k-\left(2^{*}-1\right) K^{-2}\right|+\|h-\mathcal{E}\|_{L^{\infty}(\Omega)} \leq \varepsilon_{0},
$$

where $\mathcal{E}$ denotes the Euclidean metric, then

$$
Q(\varphi, \varphi) \geq \frac{c_{1}}{2} \int_{\Omega}\left|\nabla_{h} \varphi\right|^{2} d v_{h}, \quad\left|\left\langle\varphi, e_{i}\right\rangle_{h}\right| \leq \varepsilon_{0}\|\varphi\|_{h}, \quad 0 \leq i \leq n+1,
$$

where $e_{0}=U, e_{i}=\partial U_{y, 1} /\left.\partial y^{i}\right|_{y=0}, i=1, \ldots, n, e_{n+1}=\partial U_{0, \lambda} /\left.\partial \lambda\right|_{\lambda=1}$.

Proof. We denote by $\widetilde{Q}$ the continuous bilinear form on $D^{1,2}\left(\mathbb{R}^{n}\right)$ defined by

$$
\widetilde{Q}(\widetilde{\varphi}, \widetilde{\psi})=\int_{\mathbb{R}^{n}}\left\{\nabla \widetilde{\varphi} \cdot \nabla \widetilde{\psi}-\frac{2^{*}-1}{K^{2}} U^{2^{*}-2} \widetilde{\varphi} \widetilde{\psi}\right\} d y
$$

It is well-known (and it may be verified by pull-back to the standard $n$-sphere, in stereographic projection coordinates) that there exists $c_{1}>0$ such that

$$
\widetilde{Q}(\widetilde{\varphi}, \widetilde{\varphi}) \geq c_{1} \int_{\mathbb{R}^{n}}|\nabla \widetilde{\varphi}|^{2} d y, \quad\left\langle\widetilde{\varphi}, e_{i}\right\rangle_{\mathcal{E}}=0,0 \leq i \leq n+1 .
$$

Now the claim follows by elementary considerations. Indeed, there exist unique $\mu^{j}$, $\left|\mu^{j}\right|=O\left(\varepsilon_{0}\|\varphi\|_{h}\right)$, such that $\widetilde{\varphi}:=\varphi-\mu^{j} e_{j}$ satisfies $\left\langle\widetilde{\varphi}, e_{i}\right\rangle_{\mathcal{E}}=0, \forall 0 \leq i \leq n+1$. (4.7) holds for $\widetilde{\varphi}$, and the claim follows easily.

We introduce some notations. We set

$$
\widetilde{\Omega}_{\alpha}=\mu_{\alpha}^{-1}\left(\exp _{\tilde{x}_{\alpha}}^{\widehat{g}}\right)^{-1}\left(B_{\alpha}\right) \subset \mathbb{R}^{n} .
$$

We denote by $T_{\mu_{\alpha}}$ the transformation which maps $f: B_{\alpha} \rightarrow \mathbb{R}$ into $T_{\mu_{\alpha}} f: \widetilde{\Omega}_{\alpha} \rightarrow \mathbb{R}$ defined by

$$
\left(T_{\mu_{\alpha}} f\right)(y)=\mu_{\alpha}^{(n-2) / 2} f\left(\exp _{\tilde{x}_{\alpha}}^{\widehat{g}}\left(\mu_{\alpha} y\right)\right), \quad \forall y \in \widetilde{\Omega}_{\alpha}
$$


We denote by $g_{\alpha}$ the metric on $\widetilde{\Omega}_{\alpha}$ defined by $g_{\alpha}(y)=\widehat{g}\left(\exp _{\tilde{x}_{\alpha}}^{\widehat{\widehat{g}}}\left(\mu_{\alpha} y\right)\right)$. The following transformation properties hold:

$$
\int_{B_{\alpha}} \nabla_{\widehat{g}} \varphi \cdot \nabla_{\widehat{g}} \psi d v_{\widehat{g}}=\int_{\widetilde{\Omega}_{\alpha}} \nabla_{g_{\alpha}} T_{\mu_{\alpha}} \varphi \cdot \nabla_{g_{\alpha}} T_{\mu_{\alpha}} \psi d v_{g_{\alpha}}, \quad \forall \varphi, \psi \in H_{0}^{1}\left(B_{\alpha}\right) .
$$

If $p_{1}+\cdots+p_{k}=2^{*}$, then

$$
\begin{aligned}
& \int_{B_{\alpha}}\left|\varphi_{1}\right|^{p_{1}} \cdots\left|\varphi_{k}\right|^{p_{k}} d v_{\widehat{g}}=\int_{\widetilde{\Omega}_{\alpha}}\left|T_{\mu_{\alpha}} \varphi_{1}\right|^{p_{1}} \cdots\left|T_{\mu_{\alpha}} \varphi_{k}\right|^{p_{k}} d v_{g_{\alpha}}, \\
& \forall \varphi_{1}, \ldots, \varphi_{k} \in H_{0}^{1}\left(B_{\alpha}\right) .
\end{aligned}
$$

Proof of Lemma 4.5. Observe that by the transformation properties (4.8)-(4.9) we have

$$
Q_{\alpha}(\varphi, \varphi)=\int_{\widetilde{\Omega}_{\alpha}}\left\{\left|\nabla_{g_{\alpha}} T_{\mu_{\alpha}} \varphi\right|^{2}-k_{\alpha}\left|T_{\mu_{\alpha}} \Theta\right|^{2^{*}-3} T_{\mu_{\alpha}} \Theta\left(T_{\mu_{\alpha}} \varphi\right)^{2}\right\} d v_{g_{\alpha}} .
$$

By taking $\delta_{0}$ small, we achieve $\left|g_{\alpha}-\mathcal{E}\right| \leq \varepsilon_{0}$. By taking $\alpha_{0} \gg 1$, we achieve $\left\|T_{\mu_{\alpha}} \Theta_{\alpha}-U\right\|_{L^{2^{*}\left(\widetilde{\Omega}_{\alpha}\right)}} \leq \varepsilon_{0}$ and $\left|k_{\alpha}-\left(2^{*}-1\right) K^{-2}\right| \leq \varepsilon_{0}$. It remains to check that by taking a possibly smaller $\delta_{0}$ and a possibly larger $\alpha_{0}$, we have for all $\varphi \in W_{\alpha}$ :

$$
\left|\left\langle T_{\mu_{\alpha}} \varphi, e_{i}\right\rangle_{\mathcal{E}}\right| \leq \varepsilon_{0}\left\|T_{\mu_{\alpha}} \varphi\right\|_{g_{\alpha}} .
$$

We check the above for $i=0$. Since $\varphi \in W_{\alpha}$,

$$
\begin{aligned}
0 & =\int_{B_{\alpha}} \nabla_{\widehat{g}} \varphi \cdot \nabla_{\widehat{g}} \sigma_{\alpha} d v_{\widehat{g}}=\int_{B_{\alpha}} \nabla_{\widehat{g}} \varphi \cdot \nabla_{\widehat{g}} \xi_{\alpha} d v_{\widehat{g}} \\
& =\int_{\widetilde{\Omega}_{\alpha}} \nabla_{g_{\alpha}} T_{\mu_{\alpha}} \varphi \cdot \nabla_{g_{\alpha}} T_{\mu_{\alpha}} \xi_{\alpha} d v_{g_{\alpha}}=\int_{\widetilde{\Omega}_{\alpha}} g_{\alpha}^{i j} \frac{\partial T_{\mu_{\alpha}} \varphi}{\partial y^{i}} \frac{\partial \xi_{\alpha}}{\partial y^{j}} \sqrt{\operatorname{det} g_{\alpha}} d y .
\end{aligned}
$$

Therefore:

$$
\begin{aligned}
& \int_{\widetilde{\Omega}_{\alpha}} \nabla T_{\mu_{\alpha}} \varphi \cdot \nabla U d y \mid \\
\leq & \left|\int_{\widetilde{\Omega}_{\alpha}} \nabla T_{\mu_{\alpha}} \varphi \cdot \nabla\left(U-T_{\mu_{\alpha}} \xi_{\alpha}\right) d y\right|+\left|\int_{\widetilde{\Omega}_{\alpha}}\left(\delta^{i j}-g_{\alpha}^{i j} \sqrt{\operatorname{det} g_{\alpha}}\right) \frac{\partial T_{\mu_{\alpha}} \varphi}{\partial y^{i}} \frac{\partial \xi_{\alpha}}{\partial y^{j}} d y\right| \\
\leq & \left(\left\|U-T_{\mu_{\alpha}} \xi_{\alpha}\right\|+\sup _{\widetilde{\Omega}_{\alpha}}\left|\delta^{i j}-g_{\alpha}^{i j} \sqrt{\operatorname{det} g_{\alpha}}\right|\left\|\nabla T_{\mu_{\alpha}} \xi_{\alpha}\right\|\right) \times\left\|T_{\mu_{\alpha}} \varphi\right\| \\
= & \left(\circ_{\alpha}(1)+O_{\delta_{0}}\left(\delta_{0}^{2}\right)\right)\left\|T_{\mu_{\alpha}} \varphi\right\|_{g_{\alpha}} .
\end{aligned}
$$

The remaining conditions are verified similarly. Taking into account (4.8), we conclude by Lemma 4.6 that for all $\varphi \in W_{\alpha}$,

$$
Q_{\alpha}(\varphi, \varphi) \geq \frac{c_{1}}{2} \int_{\widetilde{\Omega}_{\alpha}}\left|\nabla_{g_{\alpha}} T_{\mu_{\alpha}} \varphi\right|^{2} d v_{g_{\alpha}}=\frac{c_{1}}{2} \int_{B_{\alpha}}\left|\nabla_{\widehat{g}} \varphi\right|^{2} d v_{\widehat{g}},
$$

as asserted.

Proof of Proposition 4.1. Multiplying (4.5) by $w_{\alpha}$ and integrating over $B_{\alpha}$ we obtain:

$$
Q_{\alpha}\left(w_{\alpha}, w_{\alpha}\right)+\circ_{\alpha}\left(\left\|w_{\alpha}\right\|^{2}\right)=\int_{B_{\alpha}} f_{\alpha} w_{\alpha} d v_{\widehat{g}}
$$


By Lemma 4.5, in view of the form of $f_{\alpha}$ and recalling the orthogonality property $\int_{B_{\alpha}} w_{\alpha} \Delta_{\widehat{g}} \xi_{\alpha} d v_{\widehat{g}}=0$, we derive from the above:

$$
\begin{aligned}
\left\|w_{\alpha}\right\| \leq & C\left(\left\|\xi_{\alpha}^{2^{*}-1}+K^{2} \Delta_{\widehat{g}} \xi_{\alpha}\right\|_{2^{* \prime}}\right. \\
& \left.+\alpha\left\|u_{\alpha}\right\|_{L^{r}(M)}^{2-r}\left\|u_{\alpha}^{r-1}\right\|_{2^{* \prime}}+\mu_{\alpha}^{(n-2) / 2}\left\|\xi_{\alpha}^{2^{*}-2}\right\|_{2^{* \prime}}\right) .
\end{aligned}
$$

$\xi_{\alpha}$ satisfies

$$
-\Delta_{\widehat{g}} \xi_{\alpha}=K^{-2} \xi_{\alpha}^{2^{*}-1}+O\left(\xi_{\alpha}\right)
$$

It follows that

$$
\left\|\xi_{\alpha}^{2^{*}-1}+K^{2} \Delta_{\widehat{g}} \xi_{\alpha}\right\|_{2^{*^{\prime}}} \leq C\left\|\xi_{\alpha}\right\|_{2^{* \prime}} \leq C \mu_{\alpha}^{2}\|U\|_{2^{*^{\prime}, \mu_{\alpha}^{-1}}},
$$

where we have used $\lambda_{\alpha} \mu_{\alpha} \leq C$ in the last inequality. In order to estimate the second term, we note that the uniform estimate (3.2) implies: $u_{\alpha} \leq C \xi_{\alpha}$ in $B_{\alpha}$. Consequently,

$$
\left\|u_{\alpha}^{r-1}\right\|_{2^{* \prime}} \leq C\left(\int_{B_{\alpha}} \xi_{\tilde{x}_{\alpha}, \mu_{\alpha}^{-1}}^{(r-1) 2^{* \prime}} d v_{\widehat{g}}\right)^{1 / 2^{* \prime}} \leq C \mu_{\alpha}^{n-\frac{n-2}{2} r}\left\|U^{r-1}\right\|_{2^{* \prime}, \mu_{\alpha}^{-1}} .
$$

Similarly, we compute:

$$
\left\|\xi_{\alpha}^{2^{*}-2}\right\|_{2^{* \prime}} \leq C \mu_{\alpha}^{(n-2) / 2}\left\|U^{2^{*}-2}\right\|_{2^{*^{\prime},}, \mu_{\alpha}^{-1}}
$$

The asserted decay estimate for $\left\|w_{\alpha}\right\|$ follows.

In order to estimate $\left|\ell_{\alpha} t_{\alpha}^{*^{*}-1}-t_{\alpha} K^{-2}\right|$, in view of (4.10), we write $f_{\alpha}$ in the form:

$$
\begin{aligned}
f_{\alpha}= & \left(\ell_{\alpha} t_{\alpha}^{2^{*}-1}-t_{\alpha} K^{-2}\right) \xi_{\alpha}^{2^{*}-1}-\alpha\left\|u_{\alpha}\right\|_{L^{r}(M)}^{2-r} \psi^{1-2^{*}} u_{\alpha}^{r-1} \\
& +O\left(\xi_{\alpha}\right)+O\left(\mu_{\alpha}^{(n-2) / 2} \xi_{\alpha}^{2^{*}-2}\right) .
\end{aligned}
$$

Multiplying (4.5) by $\sigma_{\alpha}$, integrating over $B_{\alpha}$ and taking into account that $\int_{B_{\alpha}} \sigma_{\alpha} \Delta_{\widehat{g}} w_{\alpha}$ $d v_{\widehat{g}}=0$, we have:

$$
\begin{aligned}
& -k_{\alpha} \int_{B_{\alpha}}\left|\Theta_{\alpha}\right|^{2^{*}-3} \Theta_{\alpha} w_{\alpha} \sigma_{\alpha} d v_{\widehat{g}}+\int_{B_{\alpha}} b^{\prime}|\Theta|^{2^{*}-3} w_{\alpha}^{2} \sigma_{\alpha} d v_{\widehat{g}}+\int_{B_{\alpha}} b^{\prime \prime}\left|w_{\alpha}\right|^{2^{*}-1} \sigma_{\alpha} d v_{\widehat{g}} \\
= & \left(\ell_{\alpha} t_{\alpha}^{2^{*}-1}-t_{\alpha} K^{-2}\right) \int_{B_{\alpha}} \xi_{\alpha}^{2^{*}-1} \sigma_{\alpha} d v_{\widehat{g}}-\alpha\left\|u_{\alpha}\right\|_{L^{r}(M)}^{2-r} \int_{B_{\alpha}} \psi^{1-2^{*}} u_{\alpha}^{r-1} \sigma_{\alpha} d v_{\widehat{g}} \\
& +O\left(\int_{B_{\alpha}} \xi_{\alpha} \sigma_{\alpha} d v_{\widehat{g}}\right)+O\left(\mu_{\alpha}^{(n-2) / 2}\right) \int_{B_{\alpha}} \xi_{\alpha}^{2^{*}-2} \sigma_{\alpha} d v_{\widehat{g}}
\end{aligned}
$$

and thus, using $\left|w_{\alpha}\right|+\left|\Theta_{\alpha}\right|+\left|\sigma_{\alpha}\right|+u_{\alpha} \leq C \xi_{\alpha}$, we derive:

$$
\begin{array}{r}
\left|\ell_{\alpha} t_{\alpha}^{2^{*}-1}-t_{\alpha} K^{-2}\right| \int_{B_{\alpha}} \xi_{\alpha}^{2^{*}-1} \sigma_{\alpha} d v_{\widehat{g}} \leq C\left(\int_{B_{\alpha}} \xi_{\alpha}^{2^{*}-1}\left|w_{\alpha}\right| d v_{\widehat{g}}+\int_{B_{\alpha}} \xi_{\alpha}^{2} d v_{\widehat{g}}\right. \\
\left.+\alpha\left\|u_{\alpha}\right\|_{L^{r}(M)}^{2-r} \int_{B_{\alpha}} \xi_{\alpha}^{r} d v_{\widehat{g}}+\mu_{\alpha}^{(n-2) / 2} \int_{B_{\alpha}} \xi_{\alpha}^{2^{*}-1} d v_{\widehat{g}}\right) .
\end{array}
$$

In order to compare with the decay rate of $\left\|w_{\alpha}\right\|$, it is convenient to estimate as follows:

$$
\begin{gathered}
\int_{B_{\alpha}} \xi_{\alpha}^{2^{*}-1}\left|w_{\alpha}\right| d v_{\widehat{g}} \leq C\left\|w_{\alpha}\right\| \\
\int_{B_{\alpha}} \xi_{\alpha}^{r} d v_{\widehat{g}} \leq C\left\|\xi_{\alpha}^{r-1}\right\|_{2^{* \prime}}\left\|\xi_{\alpha}\right\|_{2^{*}} \leq C \mu_{\alpha}^{n-\frac{n-2}{2} r}\left\|U^{r-1}\right\|_{2^{* \prime}, \mu_{\alpha}^{-1}}
\end{gathered}
$$




$$
\begin{gathered}
\mu_{\alpha}^{(n-2) / 2} \int_{B_{\alpha}} \xi_{\alpha}^{2^{*}-1} d v_{\widehat{g}}=\mu_{\alpha}^{(n-2) / 2}\left\|\xi_{\alpha}^{2^{*}-2}\right\|_{2^{* \prime}}\left\|\xi_{\alpha}\right\|_{2^{*}} \leq C \mu_{\alpha}^{n-2}\left\|U^{2^{*}-2}\right\|_{2^{*^{\prime}, \mu_{\alpha}^{-1}}} \\
\int_{B_{\alpha}} \xi_{\alpha}^{2} d v_{\widehat{g}} \leq C\left\|\xi_{\alpha}\right\|_{2^{*}}\left\|\xi_{\alpha}\right\|_{2^{* \prime}} \leq C \mu_{\alpha}^{2}\|U\|_{2^{* \prime}, \mu_{\alpha}^{-1}}
\end{gathered}
$$

On the other hand,

$$
\begin{aligned}
\left|\ell_{\alpha} t_{\alpha}^{2^{*}-1}-t_{\alpha} K^{-2}\right| \int_{B_{\alpha}} \xi_{\alpha}^{2^{*}-1} \sigma_{\alpha} d v_{\widehat{g}} & \geq\left|\ell_{\alpha} t_{\alpha}^{2^{*}-1}-t_{\alpha} K^{-2}\right|\left(\int_{B_{\alpha}} \xi_{\alpha}^{2^{*}} d v_{\widehat{g}}+O\left(\mu_{\alpha}^{n-2}\right)\right) \\
& \geq C^{-1}\left|\ell_{\alpha} t_{\alpha}^{2^{*}-1}-t_{\alpha} K^{-2}\right|+O\left(\mu_{\alpha}^{n-2}\right) .
\end{aligned}
$$

The estimate for $\left|\ell_{\alpha} t_{\alpha}^{2^{*}-1}-t_{\alpha} K^{-2}\right|$ is established.

5. Lower Bound for $Y_{g}$ and Proof of Theorem 1.1 for $n \geq 7$. In this section we shall carefully exploit orthogonality in order to derive a lower bound for $Y_{g}\left(u_{\alpha}\right)$, as in Proposition 5.1 below. Together with the estimates from the previous sections, it will readily imply the proof of Theorem 1.1 in the case $n \geq 7$. We shall need an $L^{2}$-estimate of $\left|\nabla_{g} u_{\alpha}\right|$ on $\partial B_{\alpha}$. This can be achieved by selecting a suitable "good radius" $\delta_{\alpha} \in\left[\delta_{0} / 2, \delta_{0}\right]$, see Lemma 5.3 below. Here is where we fix $\delta_{\alpha}$. Unless otherwise stated, we assume $n \geq 3$.

The main step towards obtaining a contradiction is the following lower bound for $Y_{g}\left(u_{\alpha}\right)$ :

Proposition 5.1 (Lower bound for $Y_{g}$ ). Let $\delta_{\alpha}$ be a "good radius". Then, for all $\alpha$ sufficiently large,

$$
Y_{g}\left(u_{\alpha}\right) \geq Y_{\widehat{g}}\left(\widetilde{\xi}_{\tilde{x}_{\alpha}, \lambda_{\alpha}}^{g_{1}}\right)+O\left(\mu_{\alpha}^{2}\|U\|_{2^{* \prime}, \mu_{\alpha}^{-1}}\left\|w_{\alpha}\right\|+\mu_{\alpha}^{n-2}\right) .
$$

Proposition 5.1 readily implies:

Corollary 5.2. The following estimates hold:

$$
\begin{array}{rl}
\alpha\left\|u_{\alpha}\right\|_{L^{r}(M)}^{2} \leq C & C\left(\mu_{\alpha}^{2}\|U\|_{2^{*^{\prime}, \mu_{\alpha}^{-1}}}+\mu_{\alpha}^{n-2}\right)+\left|K^{-2}-Y_{\widehat{g}}\left(\widetilde{\xi}_{\tilde{x}_{\alpha}, \lambda_{\alpha}}^{\underline{g}}\right)\right| \\
\alpha\left\|u_{\alpha}\right\|_{L^{r}(M)}^{2} \leq C & \left(\mu_{\alpha}^{4}\|U\|_{2^{* \prime}, \mu_{\alpha}^{-1}}^{2}+\varepsilon_{\alpha} \mu_{\alpha}^{2}\|U\|_{2^{* \prime}, \mu_{\alpha}^{-1}}\left\|U^{r-1}\right\|_{2^{* \prime}, \mu_{\alpha}^{-1}}\right. \\
& \left.+\mu_{\alpha}^{n}\|U\|_{2^{* \prime}, \mu_{\alpha}^{-1}}\left\|U^{2^{*}-2}\right\|_{2^{*^{\prime \prime}, \mu_{\alpha}^{-1}}}+\mu_{\alpha}^{n-2}\right) \\
& +\left|K^{-2}-Y_{\widehat{g}}\left(\widetilde{\xi}_{\tilde{x}_{\alpha}, \lambda_{\alpha}}^{g}\right)\right| .
\end{array}
$$

Proof. By the initial assumption on $I_{\alpha}$, we have:

$$
K^{-2}>I_{\alpha}\left(u_{\alpha}\right)=Y_{g}\left(u_{\alpha}\right)+\alpha\left\|u_{\alpha}\right\|_{L^{r}(M)}^{2} .
$$

Therefore, the lower bound as in Proposition 5.1 implies:

$$
K^{-2}>\alpha\left\|u_{\alpha}\right\|_{L^{r}(M)}^{2}+Y_{\widehat{g}}\left(\widetilde{\xi}_{\tilde{x}_{\alpha}, \lambda_{\alpha}}^{\widehat{g}}\right)+O\left(\mu_{\alpha}^{2}\|U\|_{2^{*^{\prime}, \mu_{\alpha}^{-1}}}\left\|w_{\alpha}\right\|+\mu_{\alpha}^{n-2}\right),
$$

which in turn yields:

$$
\alpha\left\|u_{\alpha}\right\|_{L^{r}(M)}^{2} \leq\left|Y_{\widehat{g}}\left(\widetilde{\xi}_{\tilde{x}_{\alpha}, \lambda_{\alpha}}^{\widehat{g}}\right)-K^{-2}\right|+C\left(\mu_{\alpha}^{2}\|U\|_{2^{*^{\prime}, \mu_{\alpha}^{-1}}}\left\|w_{\alpha}\right\|+\mu_{\alpha}^{n-2}\right) .
$$

Using $\left\|w_{\alpha}\right\| \leq C$, we obtain (i). Using the energy estimate as in Proposition 4.1, we obtain (ii).

The proof of Proposition 5.1 relies on some boundary estimates and on consequences of orthogonality, which we proceed to derive. 
Lemma 5.3 (Choice of "good radius"). There exists $C>0$ independent of $\alpha$ such that:

$$
\int_{M \backslash B_{\delta_{0} / 2}\left(x_{\alpha}\right)}\left|\nabla_{g} u_{\alpha}\right|^{2} d v_{g} \leq C \mu_{\alpha}^{n-2} .
$$

Consequently, for every $\alpha$ we can select $\delta_{\alpha} \in\left[\delta_{0} / 2, \delta_{0}\right]$ such that on $B_{\alpha}=B_{\delta_{\alpha}}\left(x_{\alpha}\right)$ we have:

$$
\int_{\partial B_{\alpha}}\left|\nabla_{g} u_{\alpha}\right|^{2} d s_{g} \leq C \mu_{\alpha}^{n-2} .
$$

Furthermore, for such a $\delta_{\alpha}$ we have:

$$
\int_{B_{\alpha}}\left|\nabla_{\widehat{g}} \chi_{\alpha}\right|^{2} d v_{\widehat{g}} \leq C \mu_{\alpha}^{n-2} .
$$

Proof. Denote by $\eta$ a smooth cutoff function to be fixed below, satisfying $0 \leq \eta \leq 1$. Multiplying (2.2) by $\eta^{2} u_{\alpha}$ and integrating by parts on $M$ we have:

$$
\int_{M} \nabla_{g} u_{\alpha} \cdot \nabla_{g}\left(\eta^{2} u_{\alpha}\right) d v_{g} \leq-c(n) \int_{M} R_{g} \eta^{2} u_{\alpha}^{2} d v_{g}+\ell_{\alpha} \int_{M} \eta^{2} u_{\alpha}^{2^{*}} d v_{g} .
$$

It follows that:

$$
\int_{M} \eta^{2}\left|\nabla_{g} u_{\alpha}\right|^{2} d v_{g} \leq C\left(\int_{M} u_{\alpha}^{2}\left(\left|\nabla_{g} \eta\right|^{2}+\eta^{2}\right) d v_{g}+\int_{M} \eta^{2} u_{\alpha}^{2^{*}} d v_{g}\right) .
$$

Choosing $\eta$ such that $\eta \equiv 1$ in $M \backslash B_{\delta_{0}}\left(x_{\alpha}\right)$, supp $\eta \subset M \backslash B_{\delta_{0} / 2}\left(x_{\alpha}\right)$, we obtain:

$$
\int_{M \backslash B_{\delta_{0}}\left(x_{\alpha}\right)}\left|\nabla_{g} u_{\alpha}\right|^{2} d v_{g} \leq C\left(\int_{M \backslash B_{\delta_{0} / 2}\left(x_{\alpha}\right)} u_{\alpha}^{2} d v_{g}+\int_{M \backslash B_{\delta_{0} / 2}\left(x_{\alpha}\right)} u_{\alpha}^{2^{*}} d v_{g}\right) .
$$

Now the statement follows by the uniform estimate as in Proposition 3.1.

Since $u_{\alpha} \in C^{1}(M)$, we can choose $\delta_{\alpha}$ such that:

$$
\int_{\partial B_{\alpha}}\left|\nabla_{g} u_{\alpha}\right|^{2} d v_{g}=\min _{\delta \in\left[\delta_{0} / 2, \delta_{0}\right]} \int_{\partial B_{\delta}\left(x_{\alpha}\right)}\left|\nabla_{g} u_{\alpha}\right|^{2} d s_{g} \leq C \mu_{\alpha}^{n-2} .
$$

Recalling the definition of $\chi_{\alpha}$, we have by standard elliptic estimates and equivalence of $g$ and $\widehat{g}$ :

$$
\int_{\partial B_{\alpha}}\left|\nabla_{\widehat{g}} \chi_{\alpha}\right|^{2} d v_{\widehat{g}} \leq C \int_{\partial B_{\alpha}}\left\{\left|\nabla_{g} u_{\alpha}\right|^{2}+u_{\alpha}^{2}\right\} d s_{g} \leq C \mu_{\alpha}^{n-2} .
$$

Proof of Proposition 5.1. By the uniform estimate (3.2) and by Lemma 5.3, we have

$$
Y_{g}\left(u_{\alpha}\right)=\frac{\int_{B_{\alpha}}\left\{\left|\nabla_{g} u_{\alpha}\right|^{2}+c(n) R_{g} u_{\alpha}^{2}\right\} d v_{g}}{\left(\int_{B_{\alpha}} u_{\alpha}^{2^{*}} d v_{g}\right)^{2 / 2^{*}}}+O\left(\mu_{\alpha}^{n-2}\right) .
$$

By conformal invariance (1.8), together with (5.1) and (3.2),

$$
\int_{B_{\alpha}}\left\{\left|\nabla_{g} u_{\alpha}\right|^{2}+c(n) R_{g} u_{\alpha}^{2}\right\} d v_{g}=\int_{B_{\alpha}}\left|\nabla_{\widehat{g}} \frac{u_{\alpha}}{\psi}\right|^{2} d v_{\widehat{g}}+O\left(\mu_{\alpha}^{n-2}\right) .
$$

Recall from Section 4 that $u_{\alpha} / \psi=t_{\alpha} \xi_{\alpha}-t_{\alpha} h_{\alpha}+\chi_{\alpha}+w_{\alpha}$. By Lemma 4.2, (4.4), (5.2), and the fact

$$
\int_{B_{\alpha}} \nabla_{\widehat{g}} h_{\alpha} \cdot \nabla_{\widehat{g}} w_{\alpha} d v_{\widehat{g}}=0=\int_{B_{\alpha}} \nabla_{\widehat{g}} \chi_{\alpha} \cdot \nabla_{\widehat{g}} w_{\alpha} d v_{\widehat{g}}
$$


we have

$$
Y_{g}\left(u_{\alpha}\right)=F\left(w_{\alpha}\right)+O\left(\mu_{\alpha}^{n-2}\right),
$$

where

$$
F(w):=\frac{\int_{B_{\alpha}}\left|\nabla_{\widehat{g}}\left(t_{\alpha} \xi_{\alpha}+w\right)\right|^{2} d v_{\widehat{g}}}{\left(\int_{B_{\alpha}}\left|t_{\alpha} \xi_{\alpha}+w\right|^{2^{*}} d v_{\widehat{g}}\right)^{2 / 2^{*}}}, \quad w \in H_{0}^{1}\left(B_{\alpha}\right) .
$$

A Taylor expansion yields:

$$
F\left(w_{\alpha}\right)=F(0)+F^{\prime}(0) w_{\alpha}+\frac{1}{2}\left\langle F^{\prime \prime}(0) w_{\alpha}, w_{\alpha}\right\rangle+o\left(\left\|w_{\alpha}\right\|^{2}\right),
$$

where $F^{\prime}, F^{\prime \prime}$ denote Fréchet derivatives. We compute:

$$
\begin{aligned}
& F^{\prime}(0) w_{\alpha}=\frac{2}{\left(\int_{B_{\alpha}}\left(t_{\alpha} \xi_{\alpha} d v_{\widehat{g}}\right)^{2^{*}}\right)^{2 / 2^{*}}} \times \\
& \quad \times\left\{\int_{B_{\alpha}} \nabla_{\widehat{g}}\left(t_{\alpha} \xi_{\alpha}\right) \cdot \nabla_{\widehat{g}} w_{\alpha} d v_{\widehat{g}}-\frac{\int_{B_{\alpha}}\left|\nabla_{\widehat{g}} t_{\alpha} \xi_{\alpha}\right|^{2} d v_{\widehat{g}}}{\int_{B_{\alpha}}\left(t_{\alpha} \xi_{\alpha}\right)^{2 *} d v_{\widehat{g}}} \int_{B_{\alpha}}\left(t_{\alpha} \xi_{\alpha}\right)^{2^{*}-1} w_{\alpha} d v_{\widehat{g}}\right\} .
\end{aligned}
$$

By orthogonality, $\int_{B_{\alpha}} \nabla_{\widehat{g}} \xi_{\alpha} \cdot \nabla_{\widehat{g}} w_{\alpha} d v_{\widehat{g}}=0$ and by (4.10)

Hence,

$$
K^{2} \int_{B_{\alpha}} \xi_{\alpha}^{2^{*}-1} w_{\alpha} d v_{\widehat{g}}=\int_{B_{\alpha}}\left(-\Delta_{\widehat{g}} \xi_{\alpha}+O\left(\xi_{\alpha}\right)\right) w_{\alpha} d v_{\widehat{g}}=O\left(\int_{B_{\alpha}} \xi_{\alpha} w_{\alpha} d v_{\widehat{g}}\right)
$$

and consequently

$$
\left|\int_{B_{\alpha}} \xi_{\alpha}^{2^{*}-1} w_{\alpha} d v_{\widehat{g}}\right| \leq C\left\|\xi_{\alpha}\right\|_{2^{* \prime}}\left\|w_{\alpha}\right\| \leq C \mu_{\alpha}^{2}\|U\|_{2^{* \prime}, \mu_{\alpha}^{-1}}\left\|w_{\alpha}\right\|
$$

Similarly, we compute:

$$
\begin{aligned}
& \left\langle F^{\prime \prime}(0) w_{\alpha}, w_{\alpha}\right\rangle=\frac{2}{\left(\int_{B_{\alpha}}\left(t_{\alpha} \xi_{\alpha}\right)^{2^{*}}\right)^{2 / 2^{*}}} \times \\
& \quad \times\left\{\int_{B_{\alpha}}\left|\nabla_{\widehat{g}} w_{\alpha}\right|^{2} d v_{\widehat{g}}-\left(2^{*}-1\right) \frac{\int_{B_{\alpha}}\left|\nabla_{\widehat{g}} \xi_{\alpha}\right|^{2} d v_{\widehat{g}}}{\int_{B_{\alpha}} \xi_{\alpha}^{2 *} d v_{\widehat{g}}} \int_{B_{\alpha}} \xi_{\alpha}^{2^{*}-2} w_{\alpha}^{2} d v_{\widehat{g}}\right\} \\
& \quad+O\left(\int_{B_{\alpha}} \xi_{\alpha}^{2^{*}-1} w_{\alpha} d v_{\widehat{g}}\right)^{2} .
\end{aligned}
$$

By the transformations (4.8)-(4.9) and by Lemma 4.6 with

$$
\begin{aligned}
& \Omega=\widetilde{\Omega}_{\alpha}, \quad \Theta=T_{\mu_{\alpha}} \xi_{\alpha}, \quad h=g_{\alpha}, \\
& k=\left(2^{*}-1\right) \frac{\int_{B_{\alpha}}\left|\nabla_{\widehat{g}} \xi_{\alpha}\right|^{2} d v_{\widehat{g}}}{\int_{B_{\alpha}} \xi_{\alpha}^{2 *} d v_{\widehat{g}}},
\end{aligned}
$$

we obtain, for large $\alpha$, that

$$
\int_{B_{\alpha}}\left|\nabla_{\widehat{g}} w_{\alpha}\right|^{2} d v_{\widehat{g}}-\left(2^{*}-1\right) \frac{\int_{B_{\alpha}}\left|\nabla_{\widehat{g}} \xi_{\alpha}\right|^{2} d v_{\widehat{g}}}{\int_{B_{\alpha}} \xi_{\alpha}^{2 *} d v_{\widehat{g}}} \int_{B_{\alpha}} \xi_{\alpha}^{2^{*}-2} w_{\alpha}^{2} d v_{\widehat{g}} \geq \frac{c_{1}}{2}\left\|w_{\alpha}\right\|^{2} .
$$

Consequently,

$$
\left\langle F^{\prime \prime}(0) w_{\alpha}, w_{\alpha}\right\rangle \geq \frac{c_{1}}{2}\left\|w_{\alpha}\right\|^{2}+O\left(\mu_{\alpha}^{4}\right)\|U\|_{2^{* \prime}, \mu_{\alpha}^{-1}}^{2}\left\|w_{\alpha}\right\|^{2} .
$$

Inserting into (5.5) and observing that $\mu_{\alpha}^{2}\|U\|_{2^{* \prime}, \mu_{\alpha}^{-1}}=\circ_{\alpha}(1)$, we derive:

$$
F\left(w_{\alpha}\right) \geq F(0)+O\left(\mu_{\alpha}^{2}\|U\|_{2^{* \prime}, \mu_{\alpha}^{-1}}\left\|w_{\alpha}\right\|+\mu_{\alpha}^{n-2}\right) .
$$


Returning to (5.3) and taking into account that

$$
F(0)=Y_{\widehat{g}}\left(\widetilde{\xi}_{\tilde{x}_{\alpha}, \lambda_{\alpha}}^{\widetilde{g}}\right)+O\left(\mu_{\alpha}^{n-2}\right),
$$

we obtain the asserted lower bound.

Proof of Theorem 1.1 for $n \geq 7$. By straightforward computations,

$$
\left\|U^{q}\right\|_{2^{* \prime}, \mu_{\alpha}^{-1}} \leq \begin{cases}C, & \text { if } q>(n+2) /[2(n+2)], \\ \left(\log \mu_{\alpha}^{-1}\right)^{1 / 2^{* \prime}}, & \text { if } q=(n+2) /[2(n+2)], \\ \mu_{\alpha}^{-\frac{n+2}{2}+q(n-2)}, & \text { if } q<(n+2) /[2(n+2)] .\end{cases}
$$

We take $r=\bar{r}=2 n /(n+2)$. Then, since $n \geq 7$, we have:

$$
\begin{aligned}
& \|U\|_{2^{* \prime}, \mu_{\alpha}^{-1}} \leq C, \\
& \left\|U^{\bar{r}-1}\right\|_{2^{* \prime}, \mu_{\alpha}^{-1}} \leq C\left(1+\mu_{\alpha}^{-2+\beta}\right), \\
& \left\|U^{2^{*}-2}\right\|_{2^{*^{\prime},}, \mu_{\alpha}^{-1}} \leq C \mu_{\alpha}^{-(n-6) / 2},
\end{aligned}
$$

where $\beta=(n-6)(n-2) /[2(n+2)]$ is strictly positive. Hence, (ii) in Corollary 5.2 yields:

$$
\alpha\left\|u_{\alpha}\right\|_{L^{\bar{r}}(M)}^{2} \leq\left|Y_{\widehat{g}}\left(\xi_{\alpha}, B_{\alpha}\right)-K^{-2}\right|+C\left[\mu_{\alpha}^{4}+\varepsilon_{\alpha}\left(\mu_{\alpha}^{2}+\mu_{\alpha}^{\beta}\right)\right] .
$$

By (1.6),

$$
\left|Y_{\widehat{g}}\left(\widetilde{\xi}_{\tilde{x}_{\alpha}, \lambda_{\alpha}}^{\widehat{g}}\right)-K^{-2}\right| \leq C \mu_{\alpha}^{4}
$$

In view of (2.10), we derive:

$$
\alpha\left\|u_{\alpha}\right\|_{L^{\bar{r}}(M)}^{2} \leq C \mu_{\alpha}^{4}
$$

On the other hand, rescaling, we have:

$$
\left\|u_{\alpha}\right\|_{L^{\bar{r}}(M)} \geq\left\|u_{\alpha}\right\|_{L^{\bar{r}}\left(B_{\alpha}\right)} \geq C^{-1} \mu_{\alpha}^{2}\|U\|_{L^{\bar{r}}\left(B_{1}(0)\right)} \geq C^{-1} \mu_{\alpha}^{2}
$$

and inserting into (5.8) we obtain $\alpha \leq C$, a contradiction. Hence, Theorem 1.1 is established for all $n \geq 7$.

6. Proof of Theorem 1.1 for $n=6$. In order to prove Theorem 1.1 in the remaining case $n=6$ we need a uniform lower bound for $u_{\alpha}$. Indeed we shall prove:

Proposition 6.1 (Uniform lower bound). For $n=6, r=\bar{r}=3 / 2$, and any $1 / 2<\gamma<1$, there exists some constant $C>0$, which is independent of $\alpha$, such that $u_{\alpha}$ satisfies:

$$
u_{\alpha}(x) \geq C^{-1} \mu_{\alpha}^{2} \operatorname{dist}_{g}\left(x, \tilde{x}_{\alpha}\right)^{-4}, \quad \forall x \in B_{\delta_{\alpha} \mu_{\alpha}^{\gamma}}\left(x_{\alpha}\right) \backslash B_{\mu_{\alpha}}\left(x_{\alpha}\right),
$$

for all $\alpha \gg 1$.

Proof. We equivalently show that

$$
v_{\alpha}(y) \geq C^{-1}|y|^{-4}, \quad \forall y \in B_{\delta_{\alpha} \mu_{\alpha}^{\gamma-1}}(0) \backslash B_{1} .
$$

Here $v_{\alpha}$ is defined on $\Omega_{\alpha}$ as in (2.7) and (2.8). Recall the $\delta_{0} / 2 \leq \delta_{\alpha} \leq \delta_{0} .0<\delta_{0}<1$ will be small and fixed below. We define a comparison function

$$
H_{\alpha}(y)=\tau\left(\frac{\delta_{\alpha}^{4}}{|y|^{4}}-\mu_{\alpha}^{4}\right)+L \mu_{\alpha}^{2}\left(\log \frac{1}{\mu_{\alpha}}\right)^{2 / 3} \log \left(\frac{\mu_{\alpha}|y|}{\delta_{\alpha}}\right), \quad y \in \Omega_{\alpha} \backslash B_{1}
$$

where $\tau>0, L>0$ will be chosen below. 
Since $v_{\alpha} \rightarrow U$ uniformly on $\partial B_{1}$, we first fix some $0<\tau=\tau\left(\delta_{0}\right)<1$ such that $v_{\alpha} \geq H_{\alpha}$ on $\partial B_{1}$ for large $\alpha$. Since $H_{\alpha}=0$ on $\partial \Omega_{\alpha}$, we also have $v_{\alpha} \geq H_{\alpha}$ on $\partial \Omega_{\alpha}$. We know that

$$
C_{1}^{-1} \delta_{0}^{-2} \mu_{\alpha}^{2} \leq|y|^{-2} \leq 1, \quad \text { on } \Omega_{\alpha} \backslash B_{1} .
$$

Here and in the following, $C_{1}>1$ denotes some constant depending only on $(M, g)$. Setting $g_{\alpha}(y)=g\left(\exp _{x_{\alpha}}^{g}\left(\mu_{\alpha} y\right)\right)$ we have,

$$
\begin{aligned}
& \left.\left.\left|\Delta_{g_{\alpha}}\right| y\right|^{-4}\left|\leq C_{1} \mu_{\alpha}^{2}\right| y\right|^{-4} \\
& \left.\left|\Delta_{g_{\alpha}} \log \right| y|-4| y\right|^{-2} \mid \leq C_{1} \mu_{\alpha}^{2} .
\end{aligned}
$$

Hence,

$$
\Delta_{g_{\alpha}} H_{\alpha}(y) \geq 4 L \mu_{\alpha}^{2}\left(\log \frac{1}{\mu_{\alpha}}\right)^{2 / 3}|y|^{-2}-C_{1} \mu_{\alpha}^{2}|y|^{-4}-C_{1} L \mu_{\alpha}^{4}\left(\log \frac{1}{\mu_{\alpha}}\right)^{2 / 3} .
$$

Recall from Section 1 that

$$
\varepsilon_{\alpha}=\alpha \mu_{\alpha}^{n-\frac{n-2}{2} r}\left\|u_{\alpha}\right\|_{L^{r}(M)}^{2-r} \leq \alpha\left\|u_{\alpha}\right\|_{L^{r}(M)}^{2} .
$$

By (i) in Corollary 5.2,

$$
\alpha\left\|u_{\alpha}\right\|_{L^{r}(M)}^{2} \leq C_{2}\left(\mu_{\alpha}^{2}\|U\|_{2^{* \prime}, \mu_{\alpha}^{-1}}+\mu_{\alpha}^{4}\right)+\left|Y_{\widehat{g}}\left(\widetilde{\xi}_{\tilde{x}_{\alpha}, \lambda_{\alpha}}^{\widehat{g}}\right)-K^{-2}\right| .
$$

Here and in the following, $C_{2}>1$ denotes some constant independent of $\alpha$ and $L$. By the expansion (1.6),

$$
\left|Y_{\widehat{g}}\left(\widetilde{\xi}_{\tilde{x}_{\alpha}, \lambda_{\alpha}}^{\widehat{g}}\right)-K^{-2}\right| \leq C_{2} \mu_{\alpha}^{4} \log \frac{1}{\mu_{\alpha}},
$$

and, clearly,

It follows that

$$
\|U\|_{2^{* \prime}, \mu_{\alpha}^{-1}} \leq C_{2}\left(\log \frac{1}{\mu_{\alpha}}\right)^{2 / 3}
$$

$$
\alpha\left\|u_{\alpha}\right\|_{L^{r}(M)}^{2} \leq C_{2} \mu_{\alpha}^{2}\left(\log \frac{1}{\mu_{\alpha}}\right)^{2 / 3}
$$

Together with the uniform estimate from Section 2: $v_{\alpha}(y) \leq C_{2}|y|^{-4}$, we obtain

$$
\varepsilon_{\alpha} v_{\alpha}^{1 / 2} \leq C_{2} \mu_{\alpha}^{2}\left(\log \frac{1}{\mu_{\alpha}}\right)^{2 / 3}|y|^{-2}
$$

Hence, by the equation of $v_{\alpha}$, we have, on $\Omega_{\alpha} \backslash B_{1}$, that

$$
\begin{aligned}
& -\Delta_{g_{\alpha}}\left(v_{\alpha}-H_{\alpha}\right)(y) \\
\geq & \left(4 L-C_{2}\right) \mu_{\alpha}^{2}\left(\log \frac{1}{\mu_{\alpha}}\right)^{2 / 3}|y|^{-2}-C_{2} \mu_{\alpha}^{2}|y|^{-4}-C_{1} L \mu_{\alpha}^{4}\left(\log \frac{1}{\mu_{\alpha}}\right)^{2 / 3} \\
\geq & \left(4 L-C_{2}-C_{1} \delta_{0}^{2} L\right) \mu_{\alpha}^{2}\left(\log \frac{1}{\mu_{\alpha}}\right)^{2 / 3}|y|^{-2} .
\end{aligned}
$$

We first fix $\delta_{0}>0$ small $\left(C_{1} \delta_{0}<1\right)$, and then take $L$ large, we achieve, for large $\alpha$, that

$$
-\Delta_{g_{\alpha}}\left(v_{\alpha}-H_{\alpha}\right) \geq 0 \quad \text { in } \Omega_{\alpha} \backslash B_{1} .
$$

By the maximum principle,

$$
v_{\alpha} \geq H_{\alpha} \quad \text { in } \Omega_{\alpha} \backslash B_{1} .
$$

To conclude, we observe that for any fixed $1 / 2<\gamma<1$ we can find a $C>0$ such that:

$$
H_{\alpha}(y) \geq C^{-1}|y|^{-4} \quad \text { in } B_{\delta_{\alpha} \mu_{\alpha}^{\gamma-1}} \backslash B_{1} .
$$


Proof of Theorem 1.1 for $n=6$. When $n=6$ and $r=\bar{r}=3 / 2$, we have:

$$
\begin{aligned}
& \|U\|_{2^{*^{\prime \prime}, \mu_{\alpha}^{-1}}} \leq C\left(\log \frac{1}{\mu_{\alpha}}\right)^{2 / 3}, \\
& \left\|U^{r-1}\right\|_{2^{* \prime}, \mu_{\alpha}^{-1}} \leq C \mu_{\alpha}^{-2}, \\
& \left\|U^{2^{*}-2}\right\|_{2^{* \prime}, \mu_{\alpha}^{-1}}=\|U\|_{2^{*^{\prime}, \mu_{\alpha}^{-1}}} \leq C\left(\log \frac{1}{\mu_{\alpha}}\right)^{2 / 3} .
\end{aligned}
$$

We know

$$
\left|Y_{\widehat{g}}\left(\widetilde{\xi}_{\tilde{x}_{\alpha}, \lambda_{\alpha}}^{\underline{g}}\right)-K^{-2}\right| \leq C \mu_{\alpha}^{4} \log \frac{1}{\mu_{\alpha}} .
$$

Hence, (ii) in Corollary 5.2 implies:

$$
\alpha\left\|u_{\alpha}\right\|_{L^{\bar{r}}(M)}^{2} \leq C\left(\mu_{\alpha}^{4}\left(\log \frac{1}{\mu_{\alpha}}\right)^{4 / 3}+\varepsilon_{\alpha}\left(\log \frac{1}{\mu_{\alpha}}\right)^{2 / 3}+\mu_{\alpha}^{6}\left(\log \frac{1}{\mu_{\alpha}}\right)^{4 / 3}\right)
$$

From the uniform estimate (3.2) we derive:

$$
\varepsilon_{\alpha} \leq C \alpha \mu_{\alpha}^{4}\left(\log \frac{1}{\mu_{\alpha}}\right)^{1 / 3}
$$

and by Proposition 6.1 we have

$$
\left\|u_{\alpha}\right\|_{L^{\bar{r}}\left(B_{\alpha}\right)} \geq C^{-1} \mu_{\alpha}^{2}\left(\log \frac{1}{\mu_{\alpha}}\right)^{2 / 3} .
$$

Inserting into (6.2), we obtain

$$
\alpha \mu_{\alpha}^{4}\left(\log \frac{1}{\mu_{\alpha}}\right)^{4 / 3} \leq C\left(\mu_{\alpha}^{4}\left(\log \frac{1}{\mu_{\alpha}}\right)^{4 / 3}+\alpha \mu_{\alpha}^{4} \log \frac{1}{\mu_{\alpha}}\right) .
$$

Once again we obtain $\alpha \leq C$, a contradiction. Theorem 1.1 is thus established in the remaining limit case $n=6$.

7. Appendix: A Local to Global Argument. In this Appendix we provide a proof of the local to global argument used in Theorem 1.3. We adapt some ideas from [6]. Let $(M, g)$ be a smooth compact Riemannian manifold without boundary, $n \geq 3$.

Lemma 7.1. Suppose that there exist $\bar{\varepsilon}>0$ and $A_{\bar{\varepsilon}}>0$ such that

$$
\|u\|_{L^{2^{*}(M, g)}}^{2} \leq K^{2} \int_{M}\left\{\left|\nabla_{g} u\right|^{2}+c(n) R_{g} u^{2}\right\} d v_{g}+A_{\bar{\varepsilon}}\|u\|_{L^{1}(M, g)}^{2},
$$

for all $u \in H^{1}(M)$ such that $\operatorname{diam}_{g}(\operatorname{supp}) u<\bar{\varepsilon}$. Then there exists a constant $A>0$ such that

$$
\|u\|_{L^{2^{*}(M, g)}}^{2} \leq K^{2} \int_{M}\left\{\left|\nabla_{g} u\right|^{2}+c(n) R_{g} u^{2}\right\} d v_{g}+A\|u\|_{L^{1}(M, g)}^{2}, \quad \forall u \in H^{1}(M) .
$$

Proof. By contradiction. Suppose (7.2) is not true. Then by density of smooth functions in $H^{1}(M)$, for all $\alpha>0$ there exists $r_{\alpha} \in(1,2)$ such that

$$
\ell_{\alpha}:=\inf _{u \in H^{1}(M) \backslash\{0\}} \frac{\int_{M}\left\{\left|\nabla_{g} u\right|^{2}+c(n) R_{g} u^{2}\right\} d v_{g}+\alpha\|u\|_{L^{r_{\alpha}(M)}}^{2}}{\|u\|_{L^{2^{*}}(M)}^{2}}<K^{-2} .
$$


By the results in Section 2 with $r=r_{\alpha}$, there exists $u_{\alpha} \in H^{1}(M), u_{\alpha} \geq 0$, $\int_{M} u_{\alpha}^{2^{*}} d v_{g}=1$ such that $\ell_{\alpha}=I_{\alpha}\left(u_{\alpha}\right)$. Moreover, $u_{\alpha}$ satisfies the Euler-Lagrange equation:

$$
-\Delta_{g} u_{\alpha}+c(n) R_{g} u_{\alpha}+\alpha\left\|u_{\alpha}\right\|_{L^{r_{\alpha}(M)}}^{2-r_{\alpha}} u_{\alpha}^{r_{\alpha}-1}=\ell_{\alpha} u_{\alpha}^{2^{*}-1} \quad \text { on } M
$$

Denote by $x_{\alpha}$ a maximum point of $u_{\alpha}$. By Corollary $2.5, u_{\alpha}$ concentrates in energy at $x_{\alpha}$. In particular, for any fixed $\varepsilon>0$,

$$
\lim _{\alpha \rightarrow+\infty} \int_{M \backslash B_{\varepsilon}\left(x_{\alpha}\right)}\left\{\left|\nabla_{g} u_{\alpha}\right|^{2}+u_{\alpha}^{2^{*}}\right\} d v_{g}=0 .
$$

For a fixed $0<\varepsilon<\bar{\varepsilon} / 9$, denote by $\eta$ a smooth cutoff function such that $\eta \equiv 1$ in $B_{2 \varepsilon}\left(x_{\alpha}\right), \eta \equiv 0$ in $M \backslash B_{4 \varepsilon}\left(x_{\alpha}\right), 0 \leq \eta \leq 1,\left|\nabla_{g} \eta\right| \leq \varepsilon^{-1}$ in $M$. Then, by (7.1) and the Hölder inequality,

$$
\begin{aligned}
\left\|\eta u_{\alpha}\right\|_{L^{2^{*}(M, g)}}^{2} \leq & K^{2} \int_{M}\left\{\left|\nabla_{g}\left(\eta u_{\alpha}\right)\right|^{2}+c(n) R_{g}\left(\eta u_{\alpha}\right)^{2}\right\} d v_{g} \\
& +A_{\bar{\varepsilon}}\left(\operatorname{vol}_{g} M\right)^{2-2 / r_{\alpha}}\left\|\eta u_{\alpha}\right\|_{L^{r_{\alpha}(M)}}^{2},
\end{aligned}
$$

and consequently,

$$
\begin{aligned}
& \left\|u_{\alpha}\right\|_{L^{2^{*}}\left(B_{2 \varepsilon}\left(x_{\alpha}\right)\right)}^{2} \leq K^{2} \int_{M}\left\{\left|\nabla_{g} u_{\alpha}\right|^{2}+c(n) R_{g} u_{\alpha}^{2}\right\} d v_{g}+C\left\|u_{\alpha}\right\|_{L^{r_{\alpha}}(M)}^{2} \\
& +C \int_{B_{4 \varepsilon}\left(x_{\alpha}\right) \backslash B_{2 \varepsilon}\left(x_{\alpha}\right)}\left\{\left|\nabla_{g} u_{\alpha}\right|^{2}+u_{\alpha}^{2}\right\} d v_{g} .
\end{aligned}
$$

In turn, using the contradiction assumption (7.3), we have

$$
\begin{aligned}
& \left\|u_{\alpha}\right\|_{L^{2^{*}}\left(B_{2 \varepsilon}\left(x_{\alpha}\right)\right)} \leq K^{2} \ell_{\alpha}-\left(\alpha K^{2}-C\right)\left\|u_{\alpha}\right\|_{L^{r_{\alpha}}(M)}^{2} \\
& +C \int_{B_{4 \varepsilon}\left(x_{\alpha}\right) \backslash B_{2 \varepsilon}\left(x_{\alpha}\right)}\left\{\left|\nabla_{g} u_{\alpha}\right|^{2}+u_{\alpha}^{2}\right\} d v_{g} .
\end{aligned}
$$

Using the expansion

$$
\left\|u_{\alpha}\right\|_{L^{2^{*}}\left(B_{2 \varepsilon}\left(x_{\alpha}\right)\right)}^{2}=1-O(1)\left\|u_{\alpha}\right\|_{L^{2^{*}}\left(M \backslash B_{2 \varepsilon}\left(x_{\alpha}\right)\right)}^{2^{*}},
$$

and recalling that $\ell_{\alpha} K<1$, we obtain

$$
\alpha\left\|u_{\alpha}\right\|_{L^{r_{\alpha}(M)}}^{2} \leq C\left\|u_{\alpha}\right\|_{L^{2^{*}}\left(M \backslash B_{2 \varepsilon}\left(x_{\alpha}\right)\right)}^{2}+C \int_{B_{4 \varepsilon}\left(x_{\alpha}\right) \backslash B_{2 \varepsilon}\left(x_{\alpha}\right)}\left\{\left|\nabla_{g} u_{\alpha}\right|^{2}+u_{\alpha}^{2}\right\} d v_{g} .
$$

Now let $\eta$ be a cutoff function supported in $M \backslash B_{\varepsilon}\left(x_{\alpha}\right)$. Multiplying (7.4) by $\eta^{2} u_{\alpha}$ and integrating by parts, we find

$$
\int_{M} \eta^{2}\left|\nabla_{g} u_{\alpha}\right|^{2} d v_{g} \leq C \int_{\operatorname{supp} \eta}\left(u_{\alpha}^{2}+u_{\alpha}^{2^{*}}\right) d v_{g}
$$

Therefore,

$$
\alpha\left\|u_{\alpha}\right\|_{L^{r_{\alpha}(M)}}^{2} \leq C\left(\left\|u_{\alpha}\right\|_{L^{2}\left(M \backslash B_{\varepsilon}\left(x_{\alpha}\right)\right)}^{2}+\left\|u_{\alpha}\right\|_{L^{2^{*}}\left(M \backslash B_{\varepsilon}\left(x_{\alpha}\right)\right)}^{2}\right) .
$$

Finally, by Moser iterations,

$$
\left\|u_{\alpha}\right\|_{L^{\infty}\left(M \backslash B_{\varepsilon}\right)} \leq C\left\|u_{\alpha}\right\|_{L^{1}(M)} \leq C\left(\operatorname{vol}_{g} M\right)^{1-1 / r_{\alpha}}\left\|u_{\alpha}\right\|_{L^{r_{\alpha}(M)}},
$$

see Corollary 2.6. The estimates (7.5)-(7.6) imply $\alpha \leq C$, a contradiction, and (7.2) is established. 
Acknowledgments. The second author is grateful to the Rutgers University Mathematics Department for hospitality, during her leave of absence from Università di Napoli Federico II.

\section{REFERENCES}

[1] Adimurthi and S.L. Yadava, Some remarks on Sobolev type inequalities, Calc. Var., 2 (1994), 427-442.

[2] F.V. Atkinson and L.A. Peletier, Elliptic equations with nearly critical growth, J. Diff. Eq., 70 (1987), 349-365.

[3] T. Aubin, Problèmes isopérimétriques et espaces de Sobolev, J. Diff. Geom., 11 (1976), 573598.

[4] T. Aubin, Équations différentielles non linéaires et problème de Yamabe concernant la courbure scalaire, J. Math. Pures Appl., 55 (1976), 269-296.

[5] T. Aubin, "Some Nonlinear Problems in Riemannian Geometry", Springer-Verlag, New York, Berlin, 1998.

[6] T. Aubin and Y.Y. Li, On the best Sobolev inequality, J. Math. Pures Appl., (1) 78 (1999), 353-387.

[7] A. Bahri and J.M. Coron, The scalar-curvature problem on standard three-dimensional sphere, J. Funct. Anal., 95 (1991), 106-172.

[8] W. Beckner, Sharp Sobolev inequalities on the sphere and the Moser-Trudinger inequality, Ann. Math., 138 (1993), 213-242.

[9] H. Brezis and E. Lieb, Sobolev inequalities with remainder terms, J. Funct. Anal., 62 (1985), 73-86.

[10] H. Brezis and L. Nirenberg, Positive solutions of nonlinear elliptic equations involving critical Sobolev exponents, Comm. Pure Appl. Math., 36 (1983), 437-477.

[11] H. Brezis and M. Marcus, Hardy's inequalities revisited, Ann. Scuola Norm. Sup. Pisa Cl. Sci., (4) 25 (1997), (1,2) (1998) 217-237.

[12] H. Brezis, M. Marcus and I. Shafrir, Extremal functions for Hardy's inequality with weight, J. Funct. Anal., 171 (2000), 177-191.

[13] H. Brezis and L.A. Peletier, Asymptotics for elliptic equations involving critical growth, in: "Partial Differential Equations and the Calculus of Variations", Vol. I, Progress in Nonlinear Differential Equations and Their Applications No. 1, Birkhäuser Boston, Boston, 1989, 149192.

[14] E. Carlen and M. Loss, On the minimization of symmetric functionals, Reviews in Mathematical Physics, Special Issue (1994), 1011-1032.

[15] L. Carleson and S.Y.A. Chang, On the existence of an extremal function for an inequality of J. Moser, Bull. Sc. Math., 110 (1986), 113-127.

[16] I. Daubechies and E. Lieb, One-electron relativistic molecules with Coulomb interaction, Comm. Math. Phys., 90 (1983), 497-510.

[17] W. Ding, J. Jost, J. Li and G. Wang, The differential equation $\Delta u=8 \pi-8 \pi e^{u}$ on a compact Riemannian surface, Asian J. Math., 1 (1997), 230-248.

[18] O. Druet, The best constants problem in Sobolev inequalities, Math. Ann., 314 (1999), 327346.

[19] O. Druet, Isoperimetric inequalities on compact manifolds, Geometria Dedicata, to appear.

[20] O. Druet, E. Hebey and M. Vaugon, Sharp Sobolev inequalities with lower order remainder terms, Trans. Amer. Math. Soc., 353 (2001), 269-289.

[21] G.F.D. Duff, "Partial Differential Equations", University of Toronto Press, Toronto, 1965.

[22] J. Escobar, Sharp constant in a Sobolev trace inequality, Indiana Univ. Math. J., 37 (1988), 687-698.

[23] L. Fontana, Sharp borderline Sobolev inequalities on compact Riemannian manifolds, Comment. Math. Helv., 68 (1993), 415-454.

[24] D. Gilbarg and N. Trudinger, "Elliptic Partial Differential Equations of Second Order", Classics in Mathematics, Springer-Verlag, Berlin/Heidelberg, 2001.

[25] H. Gillet and C. Soulé, Upper bounds for regularized determinants, Comm. Math. Phys., 199 (1998), 95-115.

[26] Z.C. Han, Asymptotic approach to singular solutions for nonlinear elliptic equations involving critical Sobolev exponent, Ann. Inst. H. Poincaré, 8 (1991), 159-174. 
[27] E. Hebey and M. Vaugon, Meilleures constantes dans le théorème d'inclusion de Sobolev, Ann. Inst. H. Poincaré, (1) 13 (1996), 57-93.

[28] J. Lee and T. Parker, The Yamabe problem, Bull. Amer. Math. Soc., 17 (1987), 37-91.

[29] Y.Y. Li and T. Ricciardi, A sharp Sobolev inequality on Riemannian manifolds, C.R. Acad. Sci. Paris, Ser. I, 335 (2002), 1-6.

[30] Y.Y. Li and M. Zhu, Sharp Sobolev trace inequalities on Riemannian manifolds with boundaries, Commun. Pure. Appl. Math., 50 (1997), 449-487.

[31] Y.Y. Li and M. Zhu, Sharp Sobolev inequalities involving boundary terms, Geom. Funct. Anal., 8 (1998), 59-87.

[32] E. Lieb, Sharp constants in the Hardy-Littlewood-Sobolev and related inequalities, Ann. of Math., 118 (1983), 349-374.

[33] E. Lieb and W. Thirring, Inequalities for the moments of the eigenvalues of the Schrödinger Hamiltonian and their relation to Sobolev inequalities, in: "Studies in Mathematical Physics", Essays in honor of Valentine Bargmann, edited by E. Lieb, B. Simon and A.S. Wightman, Princeton University Press, Princeton, New Jersey, 1976.

[34] J. Moser, A sharp form of an inequality by N. Trudinger, Indiana Math. J., 20 (1971), 10771092.

[35] M. Nolasco and G. Tarantello, On a sharp Sobolev type inequality on two dimensional compact manifolds, Arch. Rat. Mech. Anal., 145 (1998), 161-195.

[36] M. Nolasco and G. Tarantello, Double vortex condensates in the Chern-Simon-Higgs theory, Cal. Var. Partial Differential Equations, 9 (1999), 31-94.

[37] O. Rey, Proof of two conjectures of H. Brézis and L.A. Peletier, Manuscripta Math., 65 (1989), 19-37.

[38] I. Shafrir, Asymptotic behaviour of minimizing sequences for Hardy's inequality, Commun. Contemp. Math., 2 (2000), 151-189.

[39] R. Schoen, Conformal deformation of a Riemannian metric to constant scalar curvature, J. Differential Geom., 20 (1984), 479-495.

[40] R. Schoen and S.T. Yau, "Lectures on Differential Geometry", Conference Proceedings and Lecture Notes in Geometry and Topology, Volume I, International Press, Boston, 1994.

[41] G. Stampacchia, Le problème de Dirichlet pour les équations elliptiques du second ordre à coefficients discontinus, Ann. Inst. Fourier Grenoble, 15 (1965), 189-258.

[42] M. Struwe, Critical points of embeddings of $H_{0}^{1, n}$ into Orlicz spaces, Ann. Inst. H. Poincaré Anal. Non Linéaire, 5 (1988), 425-464.

[43] G. Talenti, Best constant in Sobolev inequality, Ann. Mat. Pura Appl., 110 (1976), 353-372.

[44] N. Trudinger, On imbedding into Orlicz spaces and some applications, J. Math. Mech., 17 (1967), 473-484.

[45] M. Zhu, Some general forms of sharp Sobolev inequalities, J. Funct. Anal., 156 (1998), 75-120.

[46] M. Zhu, Sharp Sobolev inequalities with interior norms, Calc. Var. Partial Differential Equations, 8 (1999), 27-43.

Final version received for publication November 2002.

E-mail address: yyli@math.rutgers.edu

E-mail address: tonia.ricciardi@unina.it 